\title{
Optimal Tuning of Thermodynamic-Based Decentralized PI Control Loops: Application to the Tennessee Eastman Process
}

\author{
Luis T. Antelo, Oliver Exler, J ulio R. Banga, Antonio A. Alonso \\ Process Engineering Group. IIM-CSIC. C/ Eduardo Cabello, 6, \\ 36208, Vigo, Spain.
}

The need of designing decentralized control loops emerges to ensure the global stability of a given process plant. To that purpose, the authors have proposed in recent works a systematic approach to derive robust decentralized controllers, which is based on the link between thermodynamics and passivity theory as well as on the fundamentals of process networks. This ThermodynamicBased Control (TBC) methodology has several steps: i) Decomposition of the considered process system into abstract mass and energy inventory networks; ii) design of conceptual mass and energy inventory control loops to guarantee the convergence of the states of the plant to a compact convex region defined by constant inventories, where input-output stability follows; iii) definition of intensive variable control loops (if needed) to achieve global stability; iv) realization of the conceptual inventory and intensive variable control loops over the available degrees of freedom in the system by using, for instance, PI controllers.

In this work, we develop a tool to tune these PI control loops, based on the solution of a nonlinear programming optimization problem (NLP), in order to complete the proposed hierarchical and systematic TBC design. The aim is to minimize a given cost function, subject to both the system dynamics as well as the 
linear and nonlinear constraints (no disturbances affecting the system are considered), where the vector of decision variables will be formed by the parameters of the PI controllers used in the defined decentralized control loops. We will test this tuning procedure in several control designs developed for the challenging benchmark of the Tennessee Eastman Process (TEP) by Ricker (1996) and Larsson et al. (2001) as well as in two TBC candidates, concluding that the best candidate among the proposed ones (in terms of final cost function) will be one of these TBC designs. For solving the NLP problem, two local (FMINCON and NOMADm) solvers and a new global (MITS) one are used, comparing their performances.

Finally, the dynamic analysis of the optimal tuned closed loop systems is camied out, finding that the presented TBC control candidates will be stable while the other control structures considered exhibit complex dynamic behaviors or even instability when disturbances affecting the process a re considered.

Keywords: Decentralized control, PI Controller, Controller Parameters, Tuning, Non Linear Programming (NLP), Tennessee Eastman Process. 


\section{Introduction}

Over the years, the area of decentralized plant-wide control has attracted the process engineering community as a challenging problem which drives continuing research efforts. The themodynamic-based control (TBC) design procedure ${ }^{1}$ tried to provide a systematic to this framework of the plant-wide control. A key step in this methodology is the formalism conceming the conceptual inventory control design, which ensures the convergence of the process states, both in tems of extensive as well as intensive va riables, to compact regions of the state space constrained by constant total mass and energy. In these sets, thermodynamics gives us a function -the entropy of the system- which has a definite curvature (concavity ${ }^{2}$ ). Moreover, the function has a well-defined maximum in those regions. Such function will be the one employed to derive natural storage and Lyapunov function candidates of use in designing controllers for stabilizing the intensive variables of the network ${ }^{3}$ (i.e. temperature, pressure and concentration).

These conceptual inventory control loops consist of linear proportionalintegral controllers for mass and energy inventories, which have to be translated into suitable control loops. This is the so called control loop realization ${ }^{1,4}$. In order to camy out this realization, the inventory control law has to be obtained as a combination of control loops implemented over the real manipulated variables available in the process. Although different control configurations could serve to that purpose, in this work we will apply PI controllers to close these loops.

These proportional integral controllers are widely used in many real chemic al plants due to their simplic ity and easy on-line re-tuning, the availability of 
a la rge number of highly effic ient, reliable and cost-effic ient commercial PI (or PID) controllers and their acceptance from the operators. In the past decades, several papers conceming the tuning of PID controllers have been developed. Some of them deal with some kind of optimal approach. The development of PID tuning rules has been one of the major areas of research conceming the PID controller. The tuning of these type of controllers involves two steps: first, initial tunings are obtained by using well-known tuning rules $^{5,6}$, for instance, Zegler-Nichols or IMCbased over a simplified model of the process.

After this, the performance of the loop is improved by taking into account the operating specifications of the process considered. This tuning refinement can be seen as an optimization procedure, where the objective will be to minimize a cost function (related with the operating cost) preserving the stability of the closed-loop system. To deal with this stability issue, several options a re possible. First, the cost function can be chosen in such a way that its minimization assures stability (which is limited by the lost of meaning that some parameters of the cost function exhibits). Other possibility is to set up the optimization problem with regard to a new parameter such that stability is guaranteed for any value of that parameter inside a given set. This is the basis of modem control approaches to linear control that a re based on the controller parametrization theory ${ }^{7,8}$.

For the concrete case of the tuning of proposed decentralized control structures for the Tennessee Eastman Process benchmark, the authors ${ }^{9-12}$ do not explicitly indicate in their woks the technique used or the way in which the tuning of the control loops is carried out. Moreover, in some cases ${ }^{10,13}$, even the values for the controller parameters are omitted to the reader. As a consequence, the 
possibility of reproducing the results presented by the authors is lost. Summarizing, the tuning of many decentralized structures developed to the TEP seems to be developed in a trial-error way, which implies a considerable effort as well as several operational problems that depends on the selected set of controller parameters since the TEP states are highly related and the plant is open loop unstable.

In this work, and in order to develop a robust and general optimization based procedure, we take advantage of the TBC design approach developed by Antelo et al. ${ }^{1,4}$. It combines concepts from thermodynamics, inventory networks and process control to construct a set of stable and robust decentralized control candidate structures. Such candidates consist of inventory control loops designed so to ensure convergence of the mass and energy inventories to given references and thus guarantee stability of the extensive properties despite plant disturbances or even parameter uncerta inty. Since the loops are defined over inventories, they contain almost every possible decentralized altemative. In this way, and by physically realizing the inventory control loops over the available degrees of freedom, the approach can be employed to construct a superstructure only containing stable (and robust) decentralized control candidates. It is on this set that NLP or MINLP problems can be defined to optimally tune the controllers and to select the best operational altema tive, respec tively.

The proposed optimal controller tuning step will complete the TBC design, being the decision vector the set of parameters (gains and time constants) corresponding to the different PI control loops defined. We will apply this tuning tool for different control designs developed by Ricker (1996), Larsson et al. (2001) 
and Antelo et al. (2007b) for the benchmark of the Tennessee Eastman process.

The paper is structured as follows: In Section 2, the Tennessee Eastman Process and the considered decentralized control structures designed to stabilize it are briefly described. The fomulation of the nonlinear optimization problem as well as the methodologies selected to solve it are presented in Section 3. In Section 4, the results obtained for the optimal tuning applied for each of the control structures described in Section 2 are presented, comparing also the performance of the optimization solvers used. Finally, the dynamic performance of the optimal closed loop systems against a set of disturbances affecting the process during an extended time horizon is discussed in Section 5. The stability issue conceming the selected control structures is a lso discussed in this section.

\section{A brief ovenview of the Tennessee Eastman process and its control}

\section{approaches}

Since the publication of the TEP example ${ }^{14}$, it has been widely used in the literature as a benchmark due to its challenging properties from a control engineering point of view: it is highly nonlinear, open-loop unstable and it presents a large number of measured and manipulated variables which offer a wide set of candidates for possible control strategies. The flow sheet for the TEP is depicted in Figure 1 . Two products ( $G$ and $H$ ) are produced from four reactants $(A, C, D$ and E). A further inert trace component (B) and one byproduct (F) are present. The process units consists of a continuous stirred tank reactor, a condenser, a flash drum and a stripper. The gaseous reactants are fed to the reactor where they are transformed into liquid products. The following reactions take place in gas phase: 


$$
\begin{aligned}
A_{(g)}+C_{(g)}+D_{(g)} & \rightarrow G_{(l)} \\
A_{(g)}+C_{(g)}+E_{(g)} & \rightarrow H_{(l)} \\
A_{(g)}+E_{(g)} & \rightarrow H_{(l)} \\
3 D_{(g)} & \rightarrow 2 F_{(l)}
\end{aligned}
$$

These reactions are irreversible and exothemic with rates that depend on temperature through Amhenius expressions, and on the reactor gas phase concentration of the reactants. The reaction heat is removed from the reactor by a cooling bundle. The products and the unreacted feeds pass through a cooler and, once condensed, enter a vapour-liquid separator. The noncondensed components recycle back to the reactor feed and the condensed ones go to a product stripper in order to remove the remaining reactants by stripping with the feed stream. Products $\mathrm{G}$ and $\mathrm{H}$ are obtained in bottoms. The inert (B) and the byproduct (F) are mainly purged from the system as a vapour from the vapourliquid separator.

As a benchmark problem, the Tennessee Eastman Process provides an opportunity for designers to propose and test their control strategies on a comparable basis. Since its first publication in 1993, researchers have attempted various control techniques on this benchmark, being the majority of the control solutions decentralized control schemes ${ }^{9-13,15,16}$, process-model based control $^{17}$, model predic tive control ${ }^{18}$ and neural network control ${ }^{19}$.

For the case of the decentra lized plant-wide control approaches, although some of the results use similar procedures, the solutions have been quite different since the control objectives were not specified in relative degrees of importance by Downs and Vogel (1993). As a consequence, researchers have tried to control certain aspects of the problem more thoroughly than others. In other words, the 
way in which they invent the control loops are drastically different due to these different researchers' interpretations of the control objectives. This have led to different selections on the pairing between the controlled variables and the degrees of freedom (first, to close the control loops and, after this, to satisfy the control objectives in the TEP). In this work, we confront two well-established control structures developed by Ricker (1996) and by Larsson et al. (2001) with the one recently proposed by Antelo et al. (2007a and 2007b) we refer here as the TBC (Thermodyna mic-Based Control) structure.

For the case of the control designs by Ricker (1996) and by Larsson et al. (2001), SIMUUNK codes are available on the TEP Archive web page developed by Prof. Lawrence Ricker. On this basis, the authors have developed the SIMULINK model for the proposed TBC design. These codes are the tools which will allow the authors to both develop and solve the NLP tuning problem as well as to validate the dynamic response of the optimal tuned control structures. A brief description of each of these selected designs is presented next.

\subsection{Selected control designs for the TEP}

The decentralized control scheme defined by Ricker (1996) operates over all operation modes defined by Downs and Vogel (1993) and is able to handle all set point changes and disturbance scenarios. The only drawbacks of this control structure are the slow response of the system to changes in the operating conditions and the complexity of the control algorithm ${ }^{12}$. The key difference between the decentralized controller of Ricker and other decentralized designs is that the production rate variable is used in ratio controllers to control all of the 
flows (as shown in the small window of Figure 2). Ricker also focused on the selection of controlled variables, suggesting to control those which for optimal cost operation should be at their constraints. The author decided to control recycle valve position (at minimum), steam valve position (at minimum), reactor level (at minimum), reactor temperature (with the reactor coolant flow) and composition of $A+C$ in reactor feed (with a ratio of the $A$ and $C$ feeds). The production rate manipulator is chosen as a combination of D and $E$. To complete the control loops acting over the reactor, he proposed to control its pressure by manipulating the purge rate. For the reactor level case, a cascade loop is developed where the reactor level set point is used to calculate the reference of the separator temperature control loop (that uses the condenser coolant flow as manipulated variable). Finally, the separator and stripper levels are controlled using the liquid outflows leaving both units. The resulting control design is as depic ted in Figure 2.

On the other hand, Larsson et al. (2001) followed up the work of Ricker (1996) on selecting controlled variables based on steady-state economics. They achieved good results by controlling, in addition to the optimally constrained variables (recycle valve position, steam valve position and agitation speed at their minimum values), the reactor temperature, the recycle flowrate (or compressor work), and the composition of $\mathrm{C}$ in purge (or in reactor feed) using the reactor coolant flowrate, the $A$ feed and the $A+C$ feed, respectively. The production rate variable is used in ratio controllers to control all of the flows while the \% of product $G$ in the product stream is controlled by a ratio of D and E feeds. The rest of the control loops involving reactor level and pressure, separator level and temperature and, stripper level are the same as those specified by Ricker (1996), as shown in 
Figure 3.

Recently, Antelo et al. (2007b) applied their systematic themodynamicbased control (TBC) design developed in a previous work ${ }^{1}$ to derive robust decentralized controllers for the Tennessee Eastman Process, ensuring the global stability of the plant. In this TBC methodology, the authors combined the results that link themodynamics with passivity theory. The basic ingridients of the theory have been established by Ydstie and Alonso in the context of passive control design $^{2}$ and control of distributed systems ${ }^{3}$. A similar line of arguments was employed by Farschman et al. ${ }^{20}$ to derive mass and energy inventory control concepts. Finally, Antelo et al. ${ }^{1}$ set the basis of exploiting the underlying algebraic structure of process networks to define a decomposition of fundamental networks into mass and energy inventory layers, over which conceptual inventory control loopscan be easily defined.

In this framework, the TEP is represented as a process network composed of coupled mass and energy inventory layers like the one depicted in Figure 4. In this Figure, each phase present in the process is represented by one circle denoting a node, and solid circles symbolize the environment (node 0). As stated in the previous work by the authors, a node is a well mixed homogeneous material region. To each node $j$ in the network, it is associated a state vector $z_{j} \in \mathbb{R}^{c+1}$ of the form:

$$
z_{j}=\left(n_{j}^{1}, \ldots, n_{j}^{c}, u_{j}\right)^{T}
$$

where $n_{j}^{k}$ represents the mole number of component $k, u_{j}$ is the intemal energy and $c$ stands for the total number of chemical species. The $\theta$ nodes forming a 
process network as well as the environment can be interconnected through: i) convective fluxes which, for every node, we refer to as $f_{j} \in \mathbb{R}^{+c}$ and $p_{j}\left(f_{j}\right) \in \mathbb{R}^{+}$ for component and energy, respectively; ii) dissipative transfer fluxes collected in vectors $\varphi^{k} \in \mathbb{R}^{+d_{c}}$ (with $k=1 \ldots, \mathrm{C}$ ) and $\psi \in \mathbb{R}^{+d_{u}}$, where $d_{c}$ and $d_{u}$ stand formass and energy dissipative transfer, respectively. In Figure 4, these dissipative fluxes are represented by solid and dashed double-head arrows for mass and energy, respectively, while convective flows are denoted by solid (mass) and dashed (energy) single a rrows.

We use this process network representation to design, for each node in both layers, conceptual mass and energy inventory control loops in order to guarantee that the states of the plant will remain on a convex invariant region, where the system will be passive, and therefore input-output sta bility can be stated.

In order to exemplify this issue, let us consider that the process network comesponding to the TEP (Figure 4) can be simplified into a new network constituted by two nodes representing the reaction and the separation parts of the process, as presented in Figure 5 for the vapor mass layer of the TEP network. The R-node represents the reaction section and the S-node the separation section, respectively. The same representation is obtained when the energy layer is considered by representing the energy inventory flows in Figure 5 by dashed arrows. As shown in Figure 5, we have five possible inventory flow candidates to close the conceptual reactor vapor mass inventory control loop: i) the feed to Rnode, ii) the feed to S-node, iii) the convective inventory flow from R-node to Snode, iv) the recycle inventory flow from S-node to R-node, and v) the outflow leaving S-node. Once this loop is established, we have four inventory flow 
altematives remaining to define the separator vapor mass inventory control. Therefore, the total number of possible candidates to camy out the realization of these conceptual mass inventories becomes $N_{\text {candidates }}=5 \cdot 4=20$. As a general rule, and whenever possible, we will use the total inventory flow leaving each node of the network. Therefore, the reactor and separator vapor mass loops will be closed by acting over the R-node and S-node outflows, respectively. The same logic applies to the liquid mass and energy layers of the TEP.

The next step in the TBC design is to realize the proposed conceptual inventory control loops by using the physical inputs-outputs of the process, since the total inventory fluxes can be the result of combining multiple convective outflow streams. As a consequence, the inventory control law has to be obtained as a combination of control loops implemented over the real manipulated variables available in the process. Finally, and due to the fact that the inventory control by itself does not ensure the convergence of these variables to a desired operation point (Antelo et al., 2007a), some extra control loops are needed to achieve the convergence of the intensive variables. In some cases, the available degrees of freedom are not enough to implement the complete control structure that ensures both extensive and intensive variables convergence to the reference values. As a consequence, the set points of the inventory controllers can be used as new manipulated variables to complete the decentralized control design.

Note that by applying the TBC approach, several stable control structure candidates can be defined. This is due to the fact that the definition and realization of the conceptual inventory control loops accepts different altematives based on the inventory flow candidates available in each layer of the network. In 
other words, the TBC approach allows the designer to develop a superstructure containing all the stable control altematives.

In this work, we have considered a subset of this superstructure involving the vapor mass layer previously presented. Inside this subset, formed by 20 conceptual altematives, two candidates are considered. The first one is the result of applying the TBC systematic design approach to the TEP, using the node outflows to control the mass inventories of the network. The resulting control structure is depicted in Figure 6 . The main difference with respect to the control design proposed by Larsson et al. (2001) is that now the reactor pressure is controlled by acting over the condenser coolant flow. By using this variable, the vapor flow leaving the reactor can be modified and then, the reactor pressure can be controlled. In addition, there exists a composition control loop of component $A$ in purge that uses as manipulated variable the set point of the separator level controller. The possibility of controlling the stripper temperature (energy inventory in this unit) can be also taken into account by manipulating the steam valve.

The second altemative considered is the TBC structure depicted in Figure 7. In this case, a new realization of the inventory control loops for the nonlinear reactor vapor mass inventory is considered by acting over the purge. This manipulated variable for the reactor pressure loop is the one proposed in the works by Ricker (1996) and Larsson et al. (2001). Finally, a new candidate to control the reactor level (D Feed) as well as an extra loop controlling the separator temperature (energy inventory) by manipulating the condenser coolant flow are defined.

Next, we present the formal statement of the optimization problem used to 
tune the PI control loops for a given process plant. This formulation will be illustrated by applying it to the particular case of the Tennessee Eastman Process.

\section{Problem statement}

Let us start pointing out that the process network dynamics obey standard conservation principles for mole number and energy ${ }^{1}$ :

$$
\begin{gathered}
\dot{n}^{k}=N_{0} f_{0}^{k}+N_{\phi} f^{k}+N_{\varphi} \varphi^{k}+\gamma W \quad n^{k}, f^{k} \in \mathbb{R}^{+\theta} ; f_{0}^{k} \in \mathbb{R}^{+d_{i}} ; k=1, \ldots, c ; \\
\dot{u}=N_{0} p_{0}+N_{\phi} p+N_{\psi} \psi+Q \quad u, p \in \mathbb{R}^{+\theta} ; p_{0} \in \mathbb{R}^{+d_{i}}
\end{gathered}
$$

with $f_{0} \in \mathbb{R}^{d_{i}}$ being the vector of extemal convective inputs (and $d_{i}$ the number of inputs), and matrices $N_{0} \in \mathbb{R}^{+\theta \times d_{i}}, N_{\phi} \in \mathbb{R}^{\theta \times \theta}, N_{\varphi} \in \mathbb{R}^{\theta \times d_{c}}$ and $N_{\psi} \in \mathbb{R}^{\theta \times d_{u}}$ describing dissipative and convective network interconnections. Finally, the extra-terms $\varepsilon \mathrm{W}$ and $Q$ are related to chemical reaction units or extemal heat sources, respectively. For the reaction tem, $\varepsilon$ and $W$ are the stoichiometric and reaction rate vectors, respectively.

By using the dissipative sub-network $\mathcal{D}\left(\theta_{j}\right)^{1}$, any process network can be viewed as the convective interconnection of $\ell$ dissipative sub-networks. Each dissipative sub-network $\mathcal{D}$ has a given component and total inventory defined as:

$$
n_{\mathcal{D}}^{k}=\sum_{i \in \mathcal{D}} n_{i}^{k} \quad n_{\mathcal{D}}=\sum_{k=1}^{c} n_{\mathcal{D}}^{k}
$$

We also introduce $\forall i \in \mathcal{D}$ the following fluxes:

$$
F_{i}=\sum_{k=1}^{c} f_{i}^{k} \quad F_{\mathcal{D}}=\sum_{i \in \mathcal{D}} F_{i}
$$

The dissipative sub-network concept allows us to consider that any process 
network has an associated inventory network, which formally can be constructed by projecting Eqns. (3)-(4) onto a set of linear operators $P_{\varphi} \in \mathbb{R}^{e \times \theta}$ and $P_{\psi} \in \mathbb{R}^{e \times \theta}$ satiffying $P_{\varphi} N_{\varphi}=0$ and $P_{\psi} N_{\psi}=0$.

$$
\begin{array}{cc}
\dot{n}_{\mathcal{I}}=\mathcal{N} F_{\mathcal{I}}+\mathcal{R} & n_{\mathcal{I}}, F_{\mathcal{I}}, \mathcal{R} \in \mathbb{R}^{+\ell} \\
\dot{u}_{\mathcal{I}}=\mathcal{N} p_{\mathcal{I}} & u_{\mathcal{I}}, p_{\mathcal{I}} \in \mathbb{R}^{+\ell}
\end{array}
$$

where $n_{\mathcal{I}}, u_{\mathcal{I}}, F_{\mathcal{I}}, p_{\mathcal{I}} \in \mathbb{R}^{+\ell}$ are the vectors of inventories and fluxes, respectively, $\mathcal{R}$ is the reaction term, and $\mathcal{N} \in \mathbb{R}^{e \ell \ell}$ is a column conservation matrix (Hangos et al., 1999), so that $1^{T} \mathcal{N}=0$.

Finally, the corresponding mass inventory network representation for a dissipative subnetwork $\mathcal{D}$ can be easily obtained by defining the following transformations:

$$
\begin{aligned}
& m_{\mathcal{D}}=\sum_{i \in \mathcal{D}} \sum_{k=1}^{c} \sigma^{k} n_{\mathcal{D}}^{k} \equiv \sum_{i \in \mathcal{D}} m_{i} \\
& \phi_{\mathcal{D}}=\sum_{i \in \mathcal{D}} \sum_{k=1}^{c} \sigma^{k} f^{k} \equiv \sum_{i \in \mathcal{D}} \phi_{i}
\end{aligned}
$$

where $\sigma^{k}$ denotes the molecular weight of component $k$, while $m_{i}$ and $\phi_{i}$ represent the hold-up and flow, in units of mass, associated to each node in the dissipative sub-network. Using relations (9) and (10), Eqn. (7) becomes:

$$
\dot{m}_{\mathcal{I}}=\mathcal{N} \phi_{\mathcal{I}} \quad m_{\mathcal{I}}, \phi_{\mathcal{I}} \in \mathbb{R}^{+\ell}
$$

For the energy layer, we have:

$$
\dot{u}_{\mathcal{I}}=\mathcal{N} p_{\mathcal{I}} \quad u_{\mathcal{I}}, p_{\mathcal{I}} \in \mathbb{R}^{+\ell}
$$

Note that the reaction term $\mathcal{R}$ in (11) disappears when projecting mole inventories onto mass inventories since the latter is a conserved property. 
In order to drive the system states to a given constant set defined by constant inventories, conceptual inventory control loops are defined by using proportional-integral c ontrollers of the form:

$$
\begin{gathered}
\phi_{\mathcal{I}}=\phi_{\mathcal{I}}^{*}+\omega_{m}\left[\left(m_{\mathcal{I}}-m_{\mathcal{I}}^{*}\right)+\frac{1}{T_{I_{m}}} \int_{0}^{t}\left(m_{\mathcal{I}}-m_{\mathcal{I}}^{*}\right)\right] d t \\
p_{\mathcal{I}}=p_{\mathcal{I}}^{*}+\omega_{u}\left[\left(u_{\mathcal{I}}-u_{\mathcal{I}}^{*}\right)+\frac{1}{T_{I_{u}}} \int_{0}^{t}\left(u_{\mathcal{I}}-u_{\mathcal{I}}^{*}\right)\right] d t
\end{gathered}
$$

where $\omega_{m}$ and $\omega_{u}$ are appropriate gains, while $T_{I_{m}}$ and $T_{I_{u}}$ are the time constants for the mass and energy layers, respectively.

At this point, let us define the general optimization problem to minimize an objective function $J$ under system dynamics and linear and/or nonlinear equality and inequality constra ints:

$$
\begin{aligned}
& \min _{v} J(z, v) \\
& \text { s.t. } \\
& f(\dot{z}, z, p, v, t)=0 \\
& h(z, p, v)=0 \\
& g(z, p, v) \geq 0 \\
& v_{l} \leq v \leq v_{u}
\end{aligned}
$$

where $z \in \mathbb{R}^{\theta \times(c+1)}$ is the matrix containing the vector of states associated to each node $\mathrm{j}$ of the network, $z$ is the time derivative of $z, p$ are a possible set of parameters characteristic of the system, $v=\left(\omega_{m}, T_{I_{m}}, \omega_{u}, T_{I_{u}}\right)$ is the vector of decision variables, where $\omega_{m}=\left(\omega_{m_{1}}, \ldots, \omega_{m_{j}}\right)^{T} \in \mathbb{R}^{\theta}, \omega_{u}=\left(\omega_{u_{1}}, \ldots, \omega_{u_{j}}\right)^{T} \in \mathbb{R}^{\theta}, T_{I_{m}}=\left(T_{I_{m_{1}}}, \ldots, T_{I_{m_{j}}}\right)^{T} \in \mathbb{R}^{\theta}$, 
$T_{I_{u}}=\left(T_{I_{u_{1}}}, \ldots, T_{I_{u_{j}}}\right)^{T} \in \mathbb{R}^{\theta}$ are the gain and time constant vectors for the mass and energy layers, respectively. $J$ is the objective function to be minimized, $f$ is the set of differential and algebraic equality constraints describing the system dynamics and $h$ and $g$ are possible equality and inequality path and/or point constraints which express additional requirements for the process performance. Lower and upper bounds restrict the search space for the decision variable vector $v$ are given with $v l$ and $v u$. Therefore, the tuning of PI controllers of a given control structure may be caried out by solving an NLP problem as the one represented in Eqn. (15).

For the concrete case of the considered TEP benchmark, the objective function proposed in the TEP definitions ${ }^{14}$ is based on the operating costs and it can be defined asfollows:

$$
\begin{gathered}
T C=P C \cdot P R+\operatorname{PrC} \cdot \operatorname{Pr} R+C C \cdot C W+S C \cdot S R \\
T C=7.5973 \frac{\$}{\mathrm{kmol}} \cdot P C+0.1434 \frac{\$}{\mathrm{kmol}} \cdot \operatorname{Pr} R+0.0536 \frac{\$}{\mathrm{kWh}} \cdot C W+0.0318 \frac{\$}{\mathrm{~kg}} \cdot S C
\end{gathered}
$$

where $T C$ are the total operating costs at the base case, $P C$ and $P R$ are the purge costs and purge flowrate, respectively. Analogously, $\operatorname{PrC}, C C$ and $S C$ are the costs associated to the product stream, compressor and steam, and $\operatorname{PrR}, C W$ and $S R$ are the product rate, the compressor work and the steam rate, respectively. Operating costs for this process a re primarily determined by the loss of raw materials (in the purge, in the product stream and by means of the two side reactions). Economic costs for the process are determined by summing the costs of the raw materials and the products leaving in the purge stream and the 
product stream, and using an assigned cost to the amount of F formed. The costs conceming the compressor work and the steam to the stripper are also included. Note that the objective function used in the NLP formulation will be the mean of these operating costs along the whole simulation time horizon. For this work, the simulation time horizon was set to $t=10 h$, as it can be considered enough time length for stabilization of the TEP. Later in this paper, the issue conceming the selection of the time horizon will be explained in more detail. Finally, note that no disturbances affecting the system a re considered in this tuning procedure.

With these considerations on the objective function, the optimal tuning problem can be represented as an NLP of the form of Eqn. (15):

$$
\begin{aligned}
& \min _{v \mathbb{R}^{36}} J(z, v)=\overline{T C} \\
& \text { s.t. } \\
& \dot{m}_{\mathcal{I}}=\mathcal{N} \phi_{\mathcal{I}} \\
& \dot{u}_{\mathcal{I}}=\mathcal{N} \hat{p}_{\mathcal{I}} \\
& g(z, p, v) \geq 0 \\
& v_{0}-0.5 \cdot v_{0} \leq v \leq v_{0}+0.5 \cdot v_{0}
\end{aligned}
$$

The lower and upperbounds for the decision variables have been set to be the $\pm 50 \%$ of the initial value for the decision vector. This value has been chosen to avoid as much as possible problems related to valve saturation. These situations have been detected in preliminary dynamic simulations when considering a value of $\pm 100 \%$ of $v_{0}$ as bounds for the decision vector.

Note that the NLP also involves the following constraints $(g(z, p, v) \geq 0)$ which are related with the reactor pressure $\left(P_{r}\right)$, temperature $\left(T_{r}\right)$ and volume $\left(V_{L_{r}}\right)$, as well as with the sepa rator and the stripper volumes $\left(V_{L_{S}}, V_{L_{v}}\right.$, respectively). 


$$
\begin{gathered}
P_{r} \leq 3000 \mathrm{Kpa} \\
2 \mathrm{~m}^{3} \leq V_{L_{r}} \leq 24 \mathrm{~m}^{3} \\
T_{r} \leq 175^{\circ} \mathrm{C} \\
1 \mathrm{~m}^{3} \leq V_{L_{s}} \leq 12 \mathrm{~m}^{3} \\
1 \mathrm{~m}^{3} \leq V_{L_{v}} \leq 6 \mathrm{~m}^{3}
\end{gathered}
$$

Note that changes in the decision variables $(v)$ may drive the system to shutdown due to the fact that one or more of the constraints defined in (18) can be violated.

\section{Solution and results}

In order to solve the problem presented in Eqn. (17), we have considered three different NLP solvers:

1. FMINCON is a local gradient-based method, implemented as a part of the MATLAB Optimization Toolbox ${ }^{\circledast}$. This solver finds as local minimum of a constrained multivariable function by means of a SQP (Sequential Quadratic Programming) algorithm. The methods uses numerical or, if available, analytical gradients.

2. NOMADm (Nonlinear Optimization for Mixed variable And DerivativesMatlab) is a $M A T L A B^{\circledR}$ code that nuns various Generalized Pattem Search (GPS) algorithms to solve nonlinear and mixed variable optimization problems. This solver ${ }^{21}$ is suitable when local gra dient-based solvers are not suitable since it does not require any derivative information to converge to a limit point that satiffies certa in optima lity conditions. However, this means that more function evaluations would nomally be used by this solver than by a derivative-based approach. It uses the called Mesh Ada ptive Direct Sea rch (MADS) algorithm, which is an extension of 
pattem search methods to nonlinearly constrained optimization problem.

3. MITS (Mixed-Integer Tabu Search) is a new global optimization algorithm ${ }^{22}$ that is based on the metaheuristic Tabu Search (TS). This methodology may handle both NLP as well as Mixed-Integer NLPs (MINLPS), being a powerful tool based on an efficient algorithm obtained by advancing the approach ${ }^{23}$ proposed by Battiti and Tecchiolli (1996). They proposed a TS algorithm that is robust for any kind of functions and self-a djusting, so that no parameters have to be set. As in many algorithms for global optimization a local solver is used to identify a local minimum by starting from an initial point and in order to reach the global minimum a special strategy for deciding where to start the local solver is applied. The local solver MISQP is a special adaptation of a sequential quadratic programming method for the mixed-integer case ${ }^{24}$ (see this reference for further information a bout this solver).

In addition, it must be pointed out that the dynamic models corresponding to the control structures by Ricker (1996) and Larsson et al. (2001) considered in this work has been obtained as SIMULINK codes from the Tennessee Eastman Challenge Archive developed by the Prof. Lawrence Ricker of the University of Washington ${ }^{\dagger}$. The dynamic model for the proposed thermodynamic-based control design has been developed also as a SIMUUNK code in the Process Engineering Group at IIM-CSIC.

The optimal values obtained for the decision variables (controller parameters) as well as the final value for the objective function achieved by the solvers for each of the considered control structures are represented in Tables 1

\footnotetext{
${ }^{\dagger}{ }^{\dagger}$ http://depts.washington.edu/control/LARRY/TE/download.html
} 
(Ricker, 1996), 2 (Larsson et al., 2001) and 3 (Antelo et al., 2007b). In these Tables, $v_{0}$ denotes the initial value for the decision vector and $v_{\text {nomad }}$ and $v_{\text {mits }}$ represent the optimal values obtained by solving the NLP problem by using NOMADm and MITS solvers, respectively (with a limit of 8,000 function evaluations). Also note that these tables are divided into two different blocks, corresponding to the gains (left part) and the time constants (right part) of the PI controllers in each loop, respectively, showing that several of the optimal controller parameters correspond to the lower or upper bounds considered previously in the NLP formulation $\left(v_{0} \pm 0.5 v_{0}\right)$. Altematively, one may consider expanding the ranges for the decision variables in order to avoid reaching the constraints. However, from a dynamic point of view, the system could exhibit saturation of several valves due to high ga in values, which is not a desirable scenario. Furthemore, the computational cost for the optimization would be prohibitive.

In addition to this tuning, we take advantage of this optimization approach to camy out a comparison both in terms of efficiency and robustness among the methodologies used to solve the NLP problem defined in Eqn.(17) for the considered control designs for the TEP. Efficiency is related to the number of function evaluations required to a mive to the solution while robustness is related to the goodness final solution ac hieved.

Regarding this last point (robustness), and looking into Table 1 corresponding to Ricker's structure, it can be shown how the optimal point obtained by MITS is almost the same that the one found by NOMADm and better than the one reached by FMINCON $(J=105.58 \$ / \mathrm{h}$ and $J=105.565 \$ / \mathrm{h}$ for MITS and NOMADm, respectively). We have reduced the cost about more than $10 \$ / \mathrm{h}$ 
compared to the initial value of $J=115.80 \$ / \mathrm{h}$, obtained when original tune of the controllers is used.

For the control design by Larsson et al. (2001), it is clearly shown in Table 2 that MIS outperforms the other two solvers, being its best point ( $J=90.508 \$ / \mathrm{h}$ ) better than the one found by NOMADm and FMINCON. We have reduced the cost about more than $32 \$ / h$ when compared to the starting point ( $J=122.724$ $\$ / \mathrm{h}$ ) and about $6.5 \$ / \mathrm{h}$ when compared to the best point found by NOMADm. From an economical point of view, this is a remarkable improvement.

Finally, for the case by Antelo et al. (2007b) the initial point of $J$ for the solvers corresponds to an objective function value $J=156.8 \$ / \mathrm{h}$. Again, MITS achieves a better point than the one found by NOMADm and FMINCON $(J=$ $147.587 \$ / \mathrm{h}$ for MITS versus $J=148.745 \$ / \mathrm{h}$ obtained by NOMADm). The reduction with respect to the starting point is more than $9 \$ / \mathrm{h}$, and about $1.2 \$ / \mathrm{h}$ when compared to the best point found by NOMADm.

Despite this, the final value of the objective function obtained by this TBC control design is greater than for the other two considered case studies. The reason for this issue is that this particular control structure uses the purge as manipulated variable to control the separator vapor mass inventory. This fact leads to high values for the purge flowrate, and as a consequence high values of the cost function defined in Eqn. (16) (where the cost tem associated to purge is the one with more weight). As a consequence, the conceptual inventory control conceming the mass inventories of the most important unit in the TEP (the reactor) was re-designed, finding a better control design candidate in tems of the objective function value. The proposed configuration, shown in Figure 7, uses D 
feed to close the reactor level control loop. In order to control the vapor mass inventory, the purge flow is used as the manipulated variable to close the reactor pressure control loop, as considered by Ricker (1996) and Larsson et al. (2001). In a general and systematic way, the optimal TBC control structure could be found among the set of candidates forming the so-called superstructure by defining and solving a complete mixed-integer nonlinear programming problem (MINLP) ${ }^{23}$. This is the scope of future work to be developed by the authors.

For the shake of clarity, only the optimal tuning parameters obtained by MITS (since for this TEP case it is revealed as the best solver among the three considered in tems of robustness as it will be shown la ter in this paper) for this new TBC candidate (Figure 7) as well as the final value of the objective function are summarized in Table 4 . The initial values of the parameters $\left(v_{0}\right)$ are the same that in Table 3. It can be stated how the final value obtained for the cost function is lower ( $J=84.289 \$ / h$ ) than the one obtained for both the initial realization carred out by the authors ( $J=147.587 \$ / \mathrm{h}$ ) as well as for Ricker's and Larsson's designs ( $J=$ $105.58 \$ / \mathrm{h}$ and $J=90.508 \$ / \mathrm{h}$, respectively). The cost reduction is specially important with respect to the cost value obtained for the initial realization ca mied out by the authors $(\approx 63 \$ / \mathrm{h}$ ). This is due to the fact that the new control structure configuration (Figure 7) results in lower purge rates, since this variable is used as manipulated variable to control the reactor pressure. For the original realization (Figure 6), large variations in the separator vapor mass inventory can be stated. The reason is that we are using the coolant flow in the condenser to control the reactor pressure, modifying the condensation rate and, therefore, the separator pressure. As a consequence, strong actions over the purge rate are needed to 
ma inta in the separator vapor mass inventory constant. This does not happen when the reactor pressure loop is closed with the purge rate.

Summanizing, and from an economic point of view, it has been demonstrated that for the considered time horizon of 10 hours, an improvement of the objective function value from the initial points was obtained by this optimal tuning procedure whatever the control structure and the methodology used to solve the NLP problem was. More precisely, MITS offers the best results in terms of robustness.

Let us consider now the effic iency analysis of the solvers. To that purpose, the convergence curves for the different solvers (Figures 8 to 10) a re constructed, showing the evolution of the best value obtained by each solver along the number of objective function evaluations (or simulations). From Figures 8 to 10, it can be concluded that the new solver MITS outperforms the other selected methods in terms of efficiency, attaining a better value of the objective function in a fewer number of function evaluations, except for the case of the structure by

Ricker (where the final value is almost the same that the one obtained by NOMADm, needing a fewer number of evaluations than MITS).

The results obtained for the PI controller parameters by applying the described optimal tuning tool have to be dynamically tested in order to analyze the stability of the optimal closed loop system. This a nalysis is shown next.

\section{Dynamic validation of the optimal tuning}

As presented previously, the objective of the proposed tuning procedure will be to solve the defined NLP problem (Eq. 15) over a set of stable control loop 
candidates. In order to check this stability issue, dynamic simulations versus a selected set of disturbances between those proposed originally ${ }^{14}$ were carried out, testing the appropriate performance of the optimal tuned control structures considered. The chosen disturbances a re:

- A random disturbance in the $A, B, C$ feed composition: IDV(8).

- A step in the condenser cooling water inlet temperature: IDV(5).

Regarding the time horizon issue, note that each evaluation of the objective function implies a simulation of the control structure considered along 10 hours. Since the maximum number of function evaluations was set to 8,000 , the selection of this time horizon was made so to avoid a inadmissible computational effort in tems of computation times. In this work, we look for results in relative admissible times. However, to test the validity of the obtained controller parameters for the different control designs considered, we will extend the time horizon until 100 hours. The idea is to check whether the optimal parameters obtained for the reduced time horizon will perform properly against disturbances for largertime periods, preserving the global stability of the plant.

Note that in order to validate the performance of the considered control structures, we will analyze the evolution along the time horizon of the production rate (i.e, the stripper underflow), the $\mathrm{G}$ and $\mathrm{H}$ product composition and the reactor pressure and temperature. These states are related with both the main control objectives established originally by Downs and Vogel (1993) (product rate and composition) as well as with the highly nonlinear reactor (reactor temperature and pressure). For the sake of simplicity, we have minimized the amount of graphical results by showing the dynamic perfomance of the best optimal tuned control 
designs (those obtained by MITS).

\subsection{Ric ker's control structure}

Starting with the original controller parameters selected by Ricker for his structure (Figure 2), the dynamic response of the process versus IDV(8) can be shown in Figure 11, where a proper control action is stated, driving the system states to their references. Now, by considering the MITS optimal parameters $\left(v_{\text {mits }}\right)$ summarized on Table 1, the response of the closed loop system for the same disturbance scenario is depicted in Figure 12. As it can be seen in the figure, an increment in the frequency and a mplitude of the peaks for the states is detected. As explained in Section 3, this behavior can be due to valve saturation when reaching their lower and upper constra ints (totally closed and/or opened). A clear illustration conceming this sa turation issue affecting Ricker's control structure will be extended later when we will consider IDV(5) as the disturbance affecting the system.

Figure 13 shows the state evolution for the original Ricker's parameters against the other selected disturbance (IDV5 - a step change in the condenser water inlet temperature). This response is smoother than the one obtained for the closed loop system versus IDV(8) (Figure 11).

The differences with respect the other disturbance scenario emerge when we consider for the control structure the optimal parameters obtained by MTS (Figure 14) since an oscillatory response affecting the system states appears. Note that the time horizon has been extended from $t=100$ hours to $t=200$ hours in order to show that the system will not shutdown despite the oscillations. The causes 
for such a complex behavior concem valve saturation ${ }^{4}$. For this concrete case, the dynamic response of the purge valve is as presented in Figure 15. An oscillatory behavior is generated by the fluctuation of the vapor mass inventory in the reactor, which defines the convex region, since the control variable considered to close this loop (the purge rate) reaches its upper and almost lower limits. Such oscillations will be transported to the whole process through the recycle rate and they are not desirable for proper plant operation.

\subsection{Control structure by Larsson et al.}

As it was made for the Ricker's structure, we analyze the dynamic performance of this control design (Figure 3) for the nominal and optimized controller parameters (summarized in Table 2). Starting with the original parameters proposed by these authors, the closed loop response under disturbance IDV(8) is presented in Figure 16, stating a proper control action. The problems arises when we consider the optimal parameters obta ined by MITS (Figure 17). It can be shown how the system destabilizes and shutdowns at $t \approx 20$ hours. This means that the control structure presented by Larsson et al. (2001) is not stable for all the search space considered for the controller parameters. The economic profit obtained by solving the NLP problem is higher than for the original parameters, but the dynamic performance of the proposed control design is not desirable since the closed loop system will be not stable.

When IDV(5) disturbance is considered, it can be shown the good performance of the closed loop system with the original parameters (Figure 18). For the MITS case, the system fails and destabilizes again (Figure 19), but the shutd own 
happens at a larger time than for the IDV(8) case.

\subsection{A TBC candidate}

Let us start, as it was made for the other structures, with the case of the closed loop system (Figure 6) with the original considered para meters acting versus IDV(8). The system states evolution is depicted in Figure 20, proving the stability for this original closed-loop system. Now, we use the MITS optimal values reflected in Table 3. The dynamic performance is shown in Figure 21. It can be checked how the optimal control structure stabilizes fast and properly the process, without the instability issues showed for this disturbance case, for instance, by the control structures by Larsson et al. (Figure 17). It must be pointed out that some peaks appears at the beginning of the simulation, corresponding to wide valves movements due to high gain values.

The responses versus IDV(5) confim that the proposed structure perform properly and ensures the global stability of the plant whatever the set of parameters used (the original one -Figure 22-, and those obtained via optimization by MITS-Figure 23).

Now, consider the improved (in tems of cost) control design candidate presented in Figure 7. For the sake of clarity, only the dynamic performance of this structure versus IDV(5) is presented (Figure 24). In this Figure, the stability of the closed-loop TEP is again stated for this sc enario.

Summarizing, and from all the dynamic tests presented, it can be concluded that the proposed control structures obtained by applying the TBC approach will ensure the global stability of the TEP plant for all the optimal tuned PI 
controllers, completing this systematic design methodology. It was also proved that the optimal tuned control structures developed Ricker and Larsson et al. exhibit several stability problems such as oscillatory phenomena due to inventory oscillations or even shutd owns of the system, respectively.

Finally, note that these non-smooth responses detected will be translated into an increment of the final value of the objective function for the extended time horizon of 100 hours considered. These values of the final objective function for $t=100$ hours are summarized in Table 5 . The values into brackets represents the variation in percentage in the final value of the objective function with respect to the value obtained when the initial parameters $\left(v_{0}\right)$ are considered for an extended time horizon of $100 \mathrm{~h}$. As shown, most of the final cost values are greater than the one obtained with the original parameters in all structures and versus all the disturbances. This is due to the fact of that the NLP solution was obtained for the defined 10 hours time range and the simulations were camied out for the extended time of 100 hours. As explained before, considering this extended time horizon to solve the NLP problem could lead to better economic results (lower than for the original case), but the computational effort will be inadmissible. However, note that for the case of existing oscillations in the dynamic responses, these will not be translated into a drastic increment of the final value of the cost since the objective function is defined as the mean of the cost along the considered time horizon and, as a consequence, the upper and lower oscillations with respect the base cost will be compensated.

\section{Conclusions}

In this contribution, a PI tuning tool based on the solution of a nonlinear 
programming optimization problem (NLP) was developed to complete the themodynamic -based control (TBC) design by Antelo et al. (2007a). The proposed tuning approach was tested over several control structure candidates designed to the challenging benchmark of the Tennessee Eastman Process. When solving this optimization problem for each of the selected control design case studies (by Ricker, 1996; Larsson et al., 2001, and two different realizations of the conceptual inventory loops derived from the TBC methodology), it can be stated that the best candidate in tems of final values of the cost function will be the TBC one depicted in Figure 7. As pointed out previously in this work, it could be possible to find a better design by solving a MINLP problem inside the superstructure of TBC candidates. This will be the a im of future research work for the authors.

When a comparasion among the solvers used to solve the tuning NLP problem is made, the new tabu search based global solver (MाS) offers the best compromise between efficiency and robustness (compared to FMINCON and NOMADm).

After the optimal tuning, dynamic tests were camied out to validate and to check if the optimal parameters a re suitable for stabilizing the system for different time horizons (the one considered for solving the NLP problem $-t=10 h$ - and an extended one $-t=100 \mathrm{~h}$ ). It is concluded that both Ricker's and Larsson's control structures exhibit complex (oscillations) or even unstable behaviors for given disturbance scenarios (IDV5 and IDV8), when extended time horizons are considered. However, the hierarchical designs derived from the TBC approach guarantee the global stability of the TEP whatever the time window, the set of controller parameters and/or the disturbance affecting the system considered. 


\section{Acknowledgements}

The authors acknowledge the financial support received from the Spanish Govemment (MCyT Projects PPQ2001-3643), Xunta de Galicia (PGIDIT02PXIC 40209PN) and "PRIMS" Marie Curie Action (MRTN-CT-2004-512233).

\section{References}

1. Antelo, L. T., Otero-Muras, I., Banga, J.R., Alonso, A. A. A systematic a pproach to plant-wide control based on themodynamics. Computers \& and Chemical Engineering. 2007a, 31(7), 677-691.

2. Ydstie, B.E., Alonso, A.A. Process systems and passivity via the Clausius-Planck inequal lity. Systems \& Control Letters, 1997, 30, 253-264.

3. Alonso, A.A., Ydstie, B.E. Stabilization of distributed systems using irreversible themodyna mic s. Automatica, 2001, 37, 1739-1755.

4. Antelo, L., Banga, J.R. \& Alonso, A.A. Hierarchical Design of Decentra lized Control Struc tures for the Tennessee Eastman Process. Submitted to Computers and Chemical Engineering, 2007b.

5. Ogunnaike, B. A. Ray, W. H. Process Dynamics, Modeling and Control, 1994, Oxford University Press, New York.

6. Skogestad, S., Postlethwaite, I. Multivariable feedback control. Analysis and design, 1996, J ohn Wiley and Sons.

7. Morani, M., Zafirou, E. Robust process control, 1989. Prentice-Hall Intemational.

8. Pedret, C., Vilanova, R., Moreno, R., Serra, I. A refinement procedure for PID control tuning. Computers \& Chemical Engineening, 2002, 26, 903-908. 
9. McAvoy, T.J ., Ye, N. Base control for The Tennessee Eastman Problem.

Computers \& Chemic al Engineering. 1994, 35(8), 130-137.

10. Lyman, P.R., Georgakis, C. Plant-wide control of de Tennessee Eastman

Problem. Computers \& Chemic al Engineering. 1995, 19, 321-331.

11. Ric ker, N.L. Decentra lized control of de Tennessee Eastman Challenge Process. J oumal of Proc ess Control. 1996, 6, 205-221.

12. Larsson, T., Skogestad, S. Self-O ptimizing control of a large-scale plant: the Tennessee Eastman process. Industrial and Chemistry Engineening Research, 2001, 40, 4889-4901.

13. Price, R.M., Lyman, P.R., Georgakis, C. Throughput manipulation in plantwide control structures. Ind. Eng. Chem. Res. 1994, 33(5), 1197-1207.

14. Downs, J. ., Vogel, E.F. A plant-wide industrial process control problem. Computers \& Chemic al Engineering. 1993, 17, 245-255.

15. Luyben, M.L., Tyreus, B.D., Luyben, W. Pla ntwide control design procedure. AIC hE J oumal. 1997, 43, 3163-3174.

16. Tyreus, B.D. Dominant variables for partial control. 2. Applic ation to the Tennessee Eastman Challenge Process. Ind. Eng. Chem. Res.1998, 38(4), 1444-1455.

17. J ockenhövel, T., Biegler, L.T., Wächter, A. Dynamic optimization of the Tennessee Eastman process using the OptC ontroIC entre. Computers \& Chemic al Engineering, 2003, 27(11), 1513-1531.

18. Ricker, N. L., Lee, J. H. Nonlinear model predic tive control of the Tennessee Eastman challenge proc ess. Computers \& Chemic al Engineering. 1995, 19(9), 961981.

19. Baughman, D.R., Lu, Y.A. Neural Networks in Bioprocessing and Chemic al 
Engineering, 1995, Academic Press, San Diego.

20. Farschman, C., Viswanath, K., Ydstie, B. Process systems and inventory control. AIChE J oumal, 1998, 44(8), 1841-1857.

21. Abramson, M. A. Pattem Search Algorithms for Mixed Variable General Constra ined Optimization Problems, 2002. Rice University, Houston, Texas.

22. Exler, O., Schittkowski K. A trust region SQP a lgorithm for mixed-integer nonlinear programming, O ptimization Letters, 2006, DOI 10.1007/s11590-006-0026-1.

23. Battiti R., Tecchiolli G. The continuous tabu search: Blending combinatorial optimization and stochastic search for global optimization. Annals of Operations Research, 1996, 63, 153-188.

24. Exler, O., Antelo, L. T., Alonso, A.A., Banga, J .R. A Tabu Search-based algorithm for integrated process and control system design, Sent to Computers \& Chemic al Eng ineering, 2006. 


\section{List of Figures}

- Figure 1. The Tennessee Eastman Process flowsheet.

- Figure 2. Control structure proposed by Ricker (1996) for the TEP.

- Figure 3. Control structure proposed by Larsson et al. (2001) for the TEP.

- Figure 4. Process network for the TEP.

- Figure 5. Simplified process network for the TEP for the va por mass layer.

- Figure 6. TBC candidate proposed by Antelo et al. (2007b) for the TEP.

- Figure 7. Altemative TBC structure obta ined for the TEP.

- Figure 8. Convergence curves for the solvers considered in the Ricker's tuning case.

- Figure 9. Convergence curves for the solvers considered in the tuning case of the.control structure by Larsson et al. (2001).

- Figure 10. Convergence curves for the solvers considered in the tuning case of the control structure by Antelo et al. (2007b).

- Figure 11. Closed loop response against IDV(8) for the control design case by Ricker (1996) (a uthor's nominal controller para meters).

- Figure 12. Closed loop response against IDV(8) for the control design case by Ricker (1996) using optima I para meters $v_{\text {mits }}$.

- Figure 13. Closed loop response against IDV(5) for the control design case by Ricker (1996) (a uthor's nominal controller para meters).

- Figure 14. Closed loop response against IDV(5) for the control design case by Ricker (1996) using optima l para meters $v_{\text {mits }}$.

- Figure 15. Purge valve position for the closed loop system (MIS optimal parameters) against IDV(5) for Ric ker's control struc ture. 
- Figure 16. Closed loop response against IDV(8) for the control design case by Larsson et al. (2001) (authors' nominal controller parameters).

- Figure 17. Closed loop response against IDV(8) for the control design case by Larsson et al. (2001) using optimal para meters $v_{\text {mits }}$.

- Figure 18. Closed loop response against IDV(5) for the control design case by Larsson et al. (2001) (authors' nominal controller parameters).

- Figure 19. Closed loop response against IDV(5) for the control design case by Larsson et al. (2001) using optimal parameters $v_{\text {mits }}$.

- Figure 20. Closed loop response against IDV(8) for the proposed TBC design (authors' nominal controller para meters).

- Figure 21. Closed loop response against IDV(8) for the proposed TBC design using optimal parameters $v_{\text {mits }}$.

- Figure 22. Closed loop response against IDV(5) for the proposed TBC design (authors' nominal controller parameters).

- Figure 23. Closed loop response against IDV(5) for the proposed TBC design using optimal parameters $v_{\text {mits }}$.

- Figure 24. Closed loop response against IDV(5) for the cost improved TBC design using optimal para meters $v_{\text {mits }}$. 


\section{List of Tables}

- Table 1. Optimal controller para meters for Ricker's control struc ture.

- Table 2. Optimal controller parameters for the control structure by Larsson et al. (2001).

- Table 3. Optimal controller parameters for the control struc ture by Antelo et al. (2007b).

- Table 4. Optimal tuning for the TBC structure (Figure 7).

- Table 5. Objective function values for all the control structures and disturbances in a time horizon of $\mathrm{t}=100 \mathrm{~h}$. 


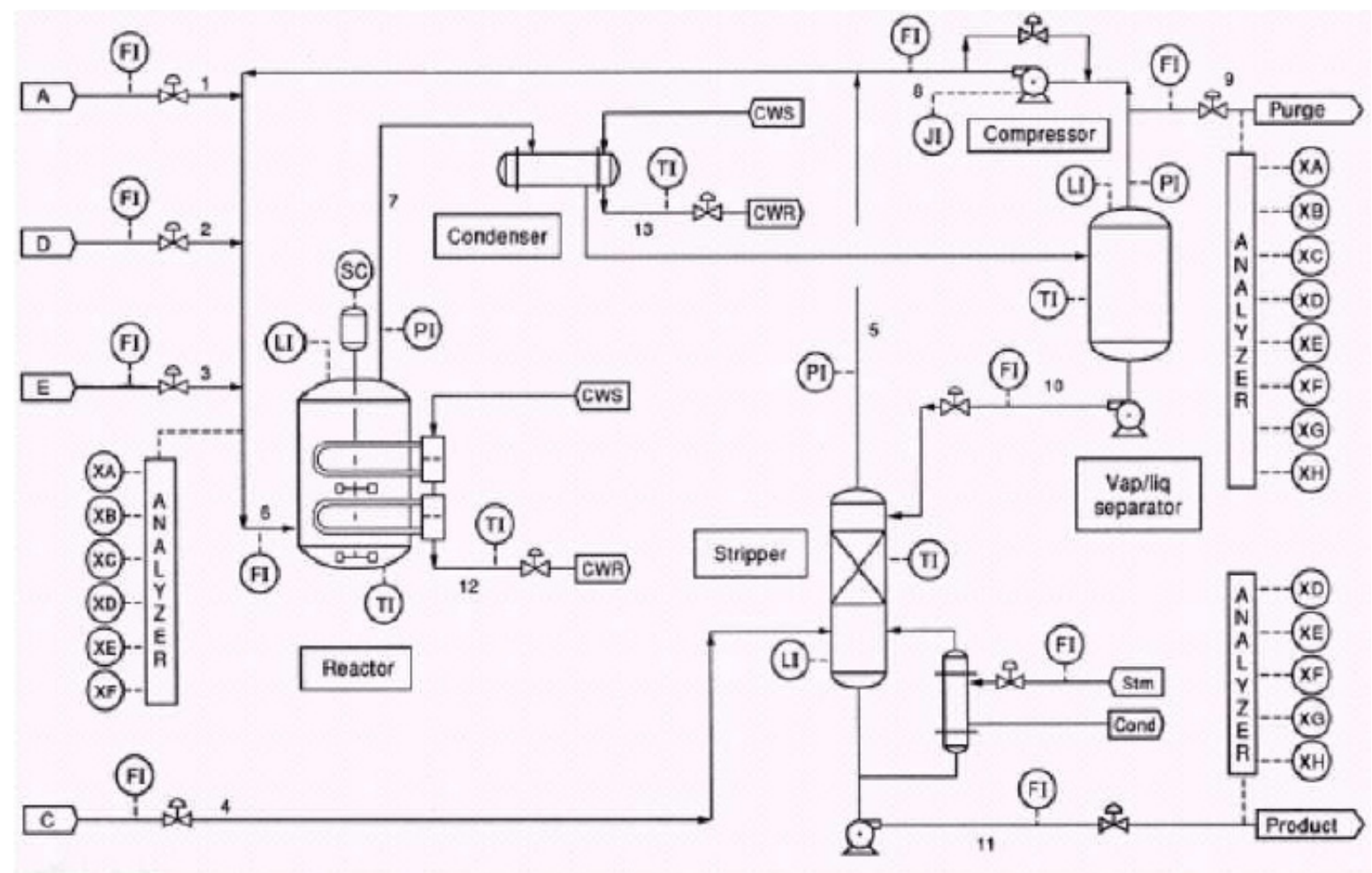

Figure 1. The Tennessee Eastman Process flowsheet. 


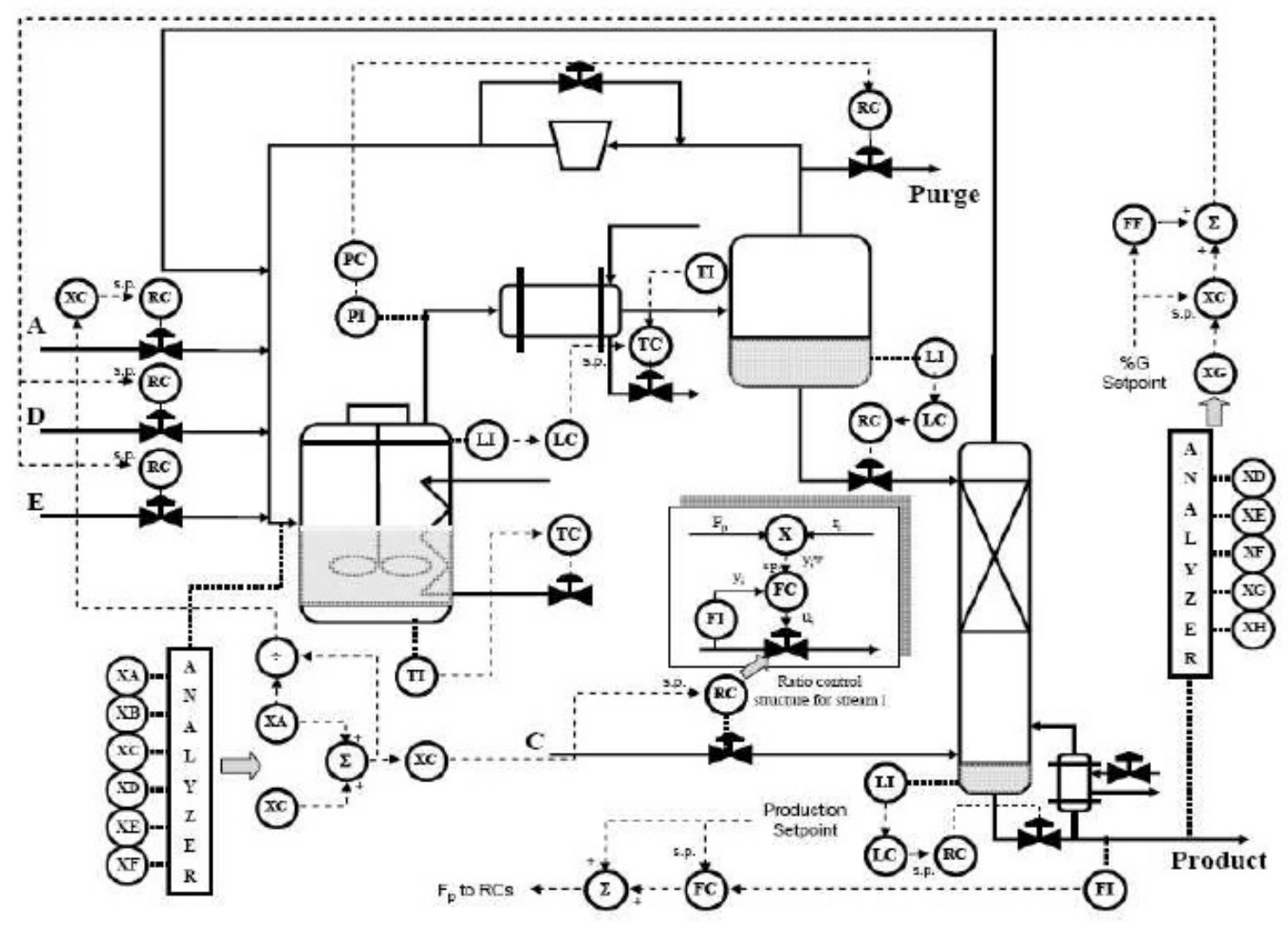

Figure 2. Control structure proposed by Ricker (1996) for the TEP. 


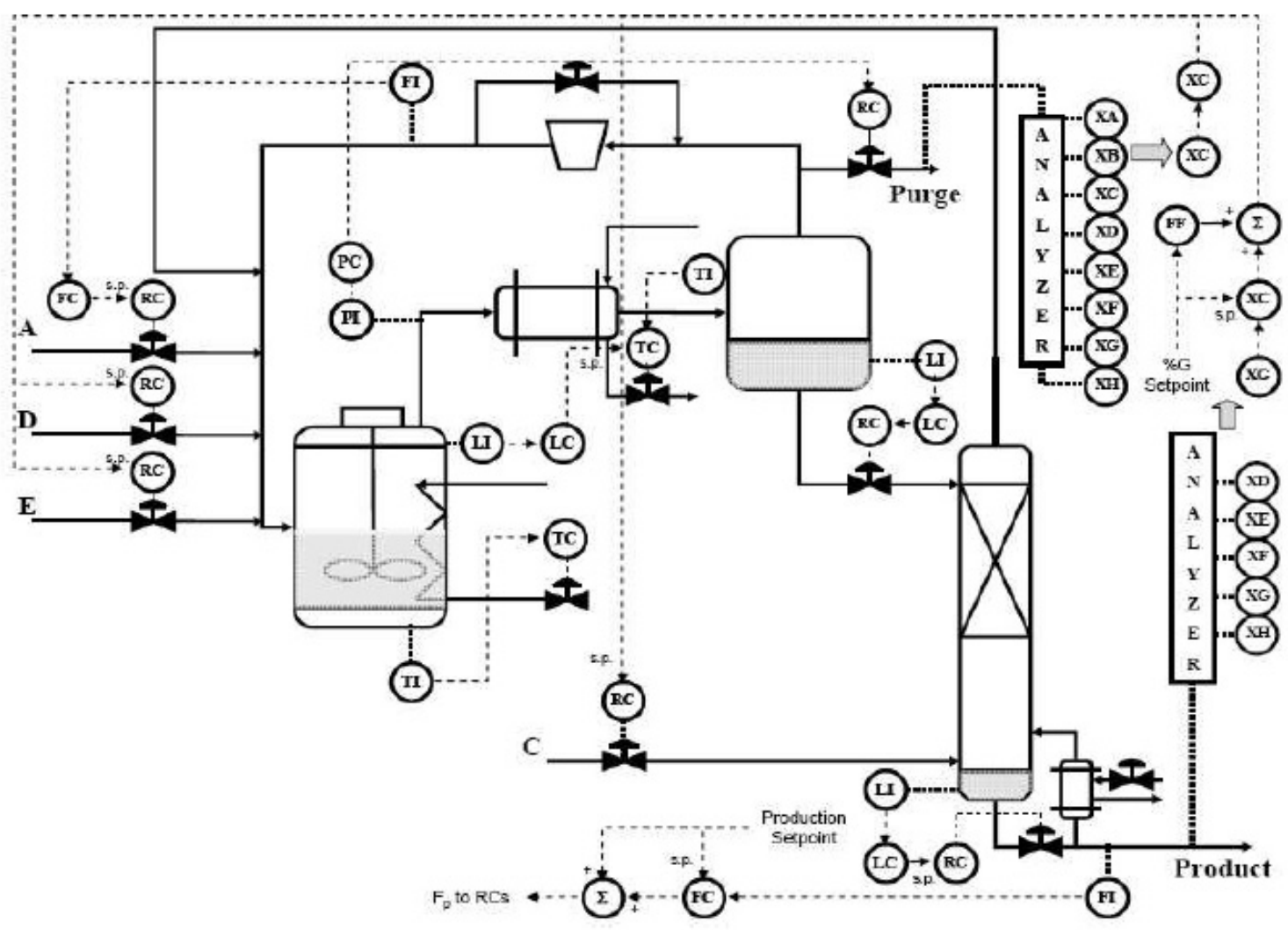

Figure 3. Control structure proposed by Larsson et al. (2001) for the TEP. 


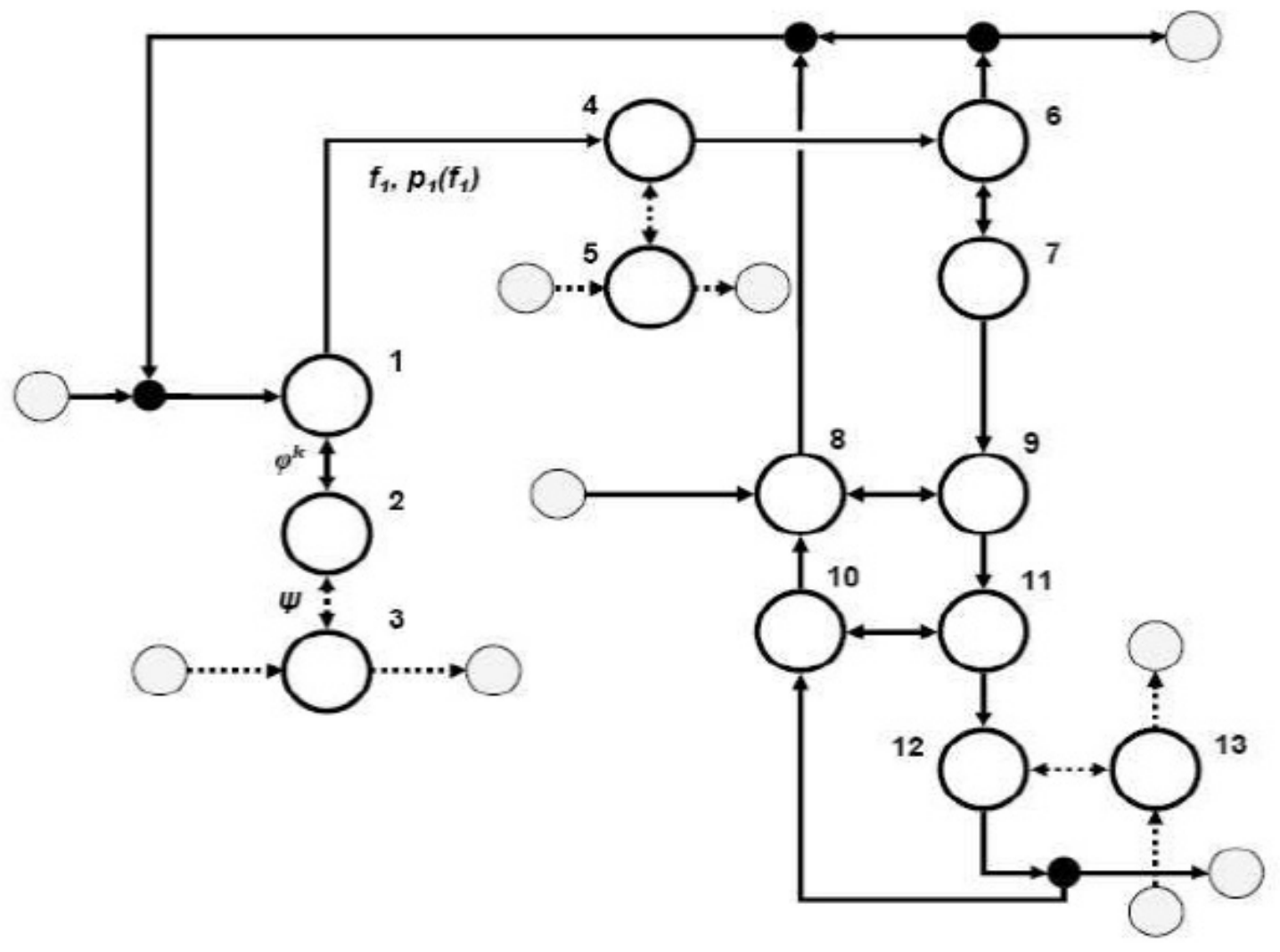

Figure 4. Process network for the TEP.

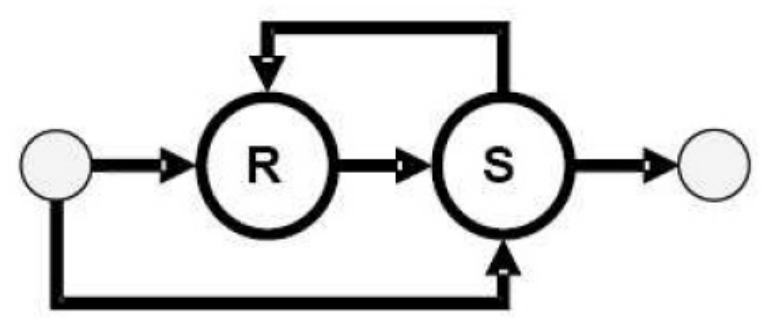

Figure 5. Simplified process network for the TEP for the va por mass layer. 


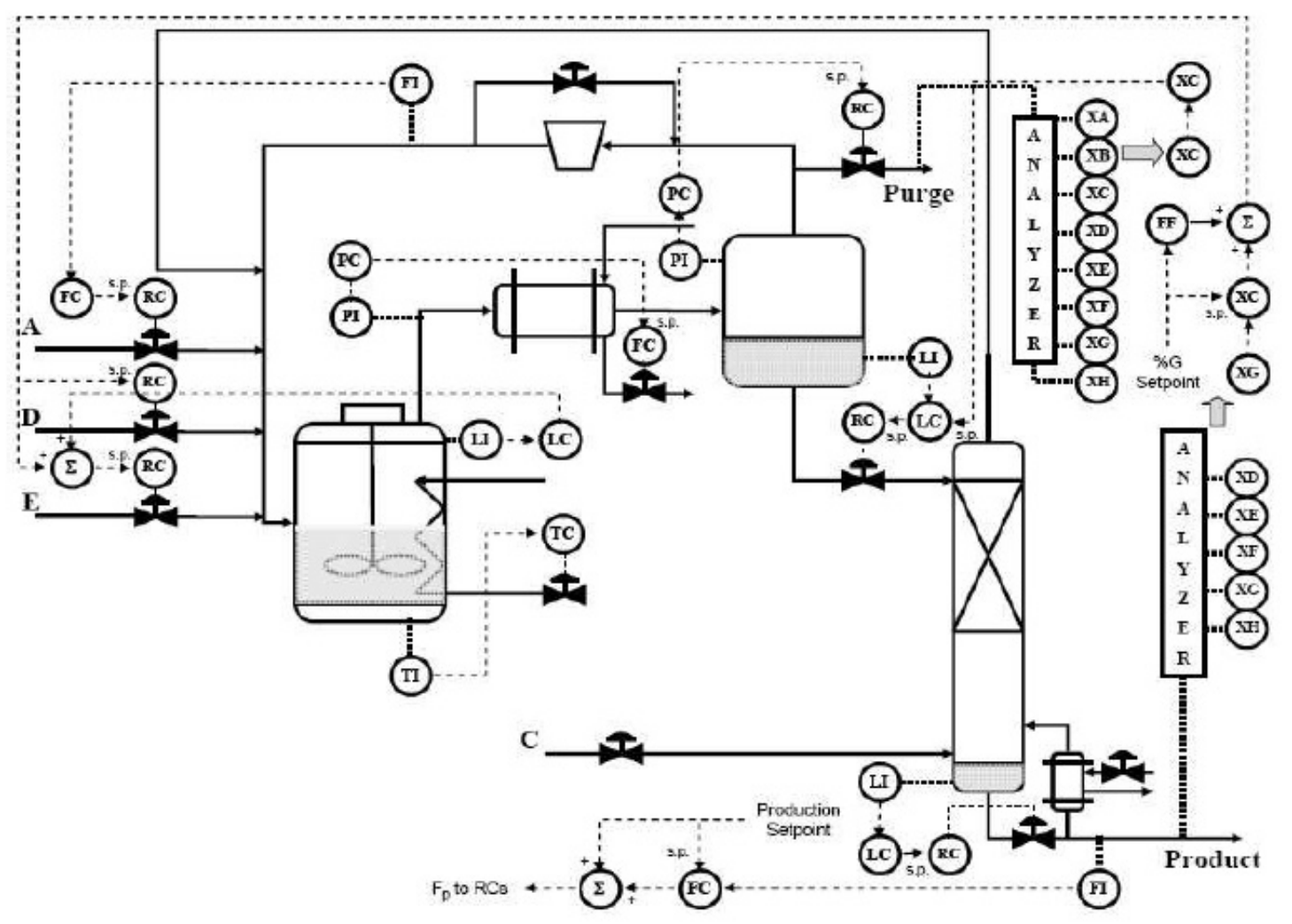

Figure 6. TBC candidate proposed by Antelo et al. (2007b) for the TEP. 


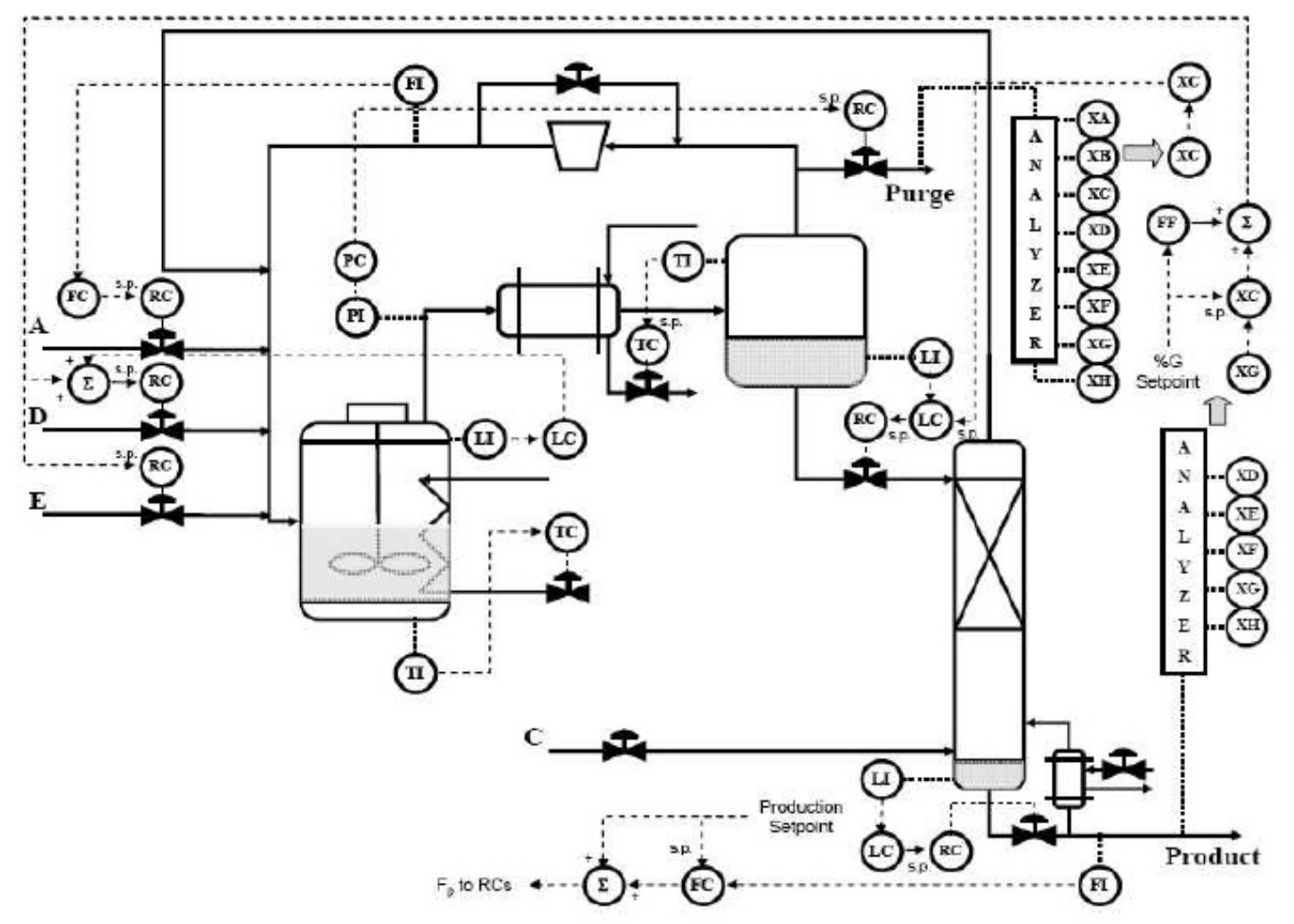

Figure 7. Altemative TBC structure obta ined for the TEP. 


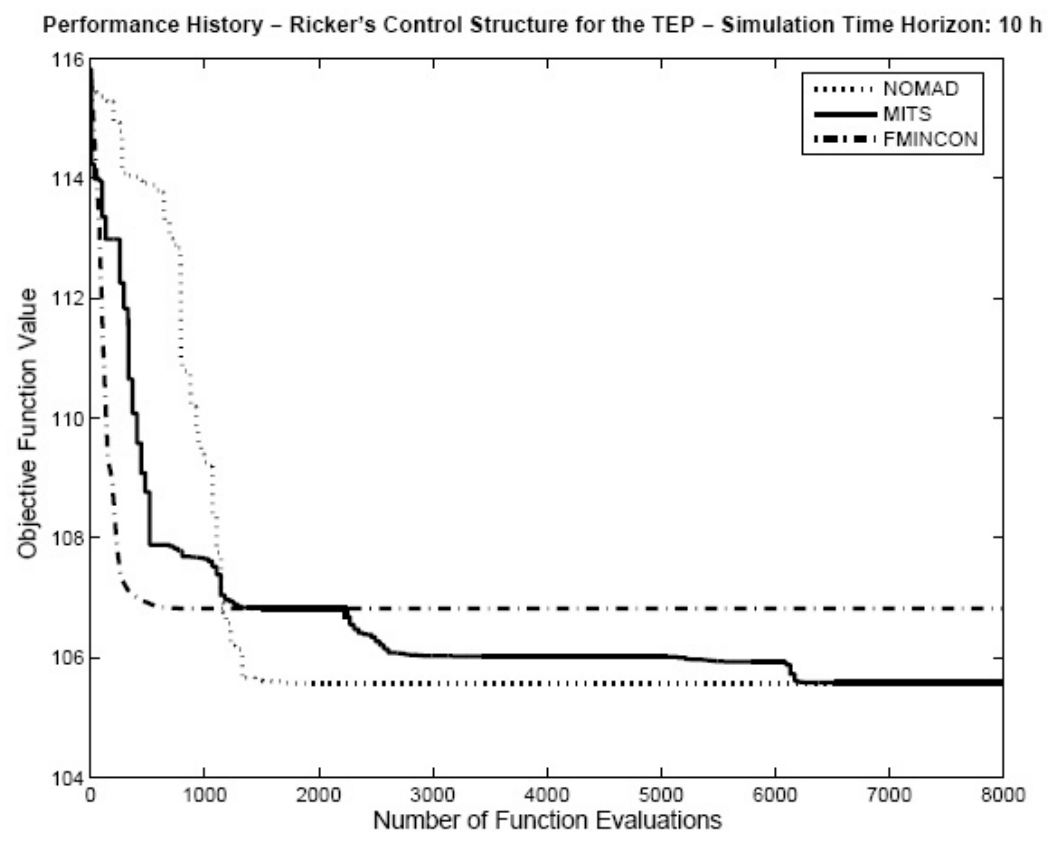

Figure 8. Convergence curves for the solvers considered in the Ricker's tuning case.

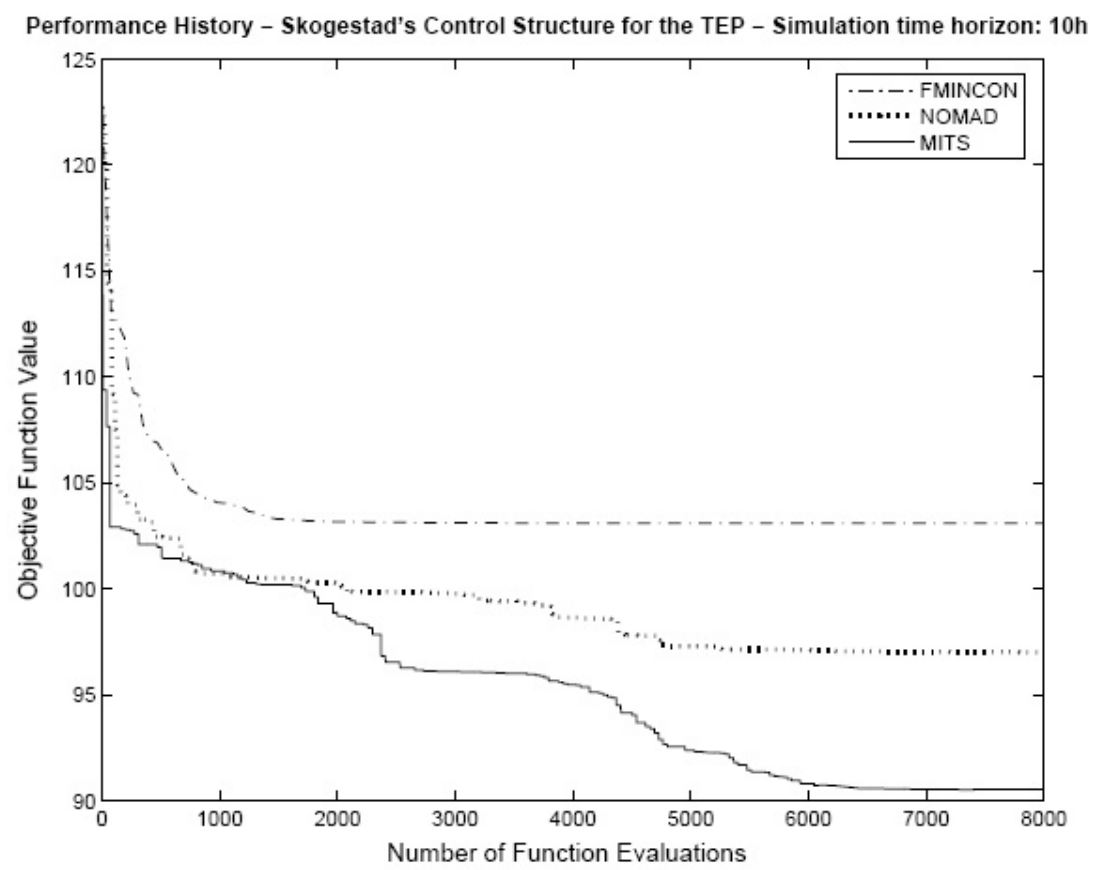

Figure 9. Convergence curves for the solvers considered in the tuning case of the.control struc ture by Larsson et al. (2001). 


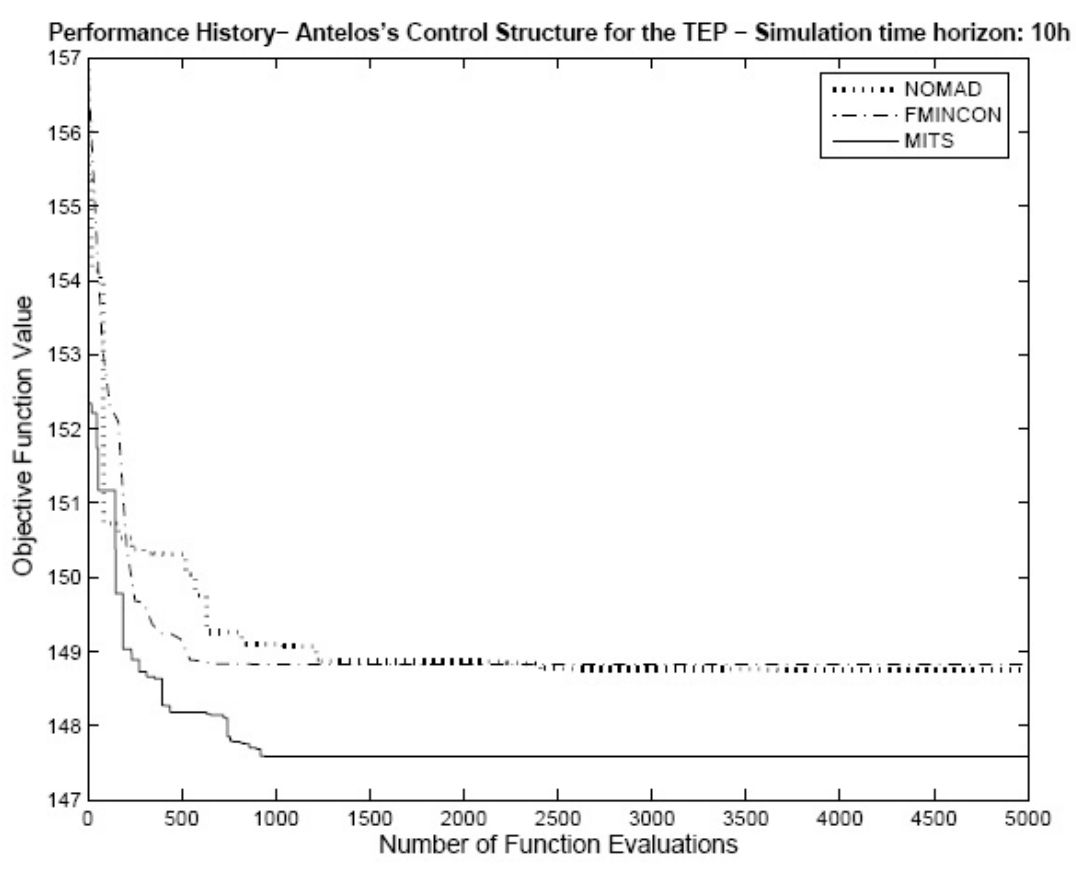

Figure 10. Convergence curves for the solvers considered in the tuning case of the control structure by Antelo et al. (2007b).
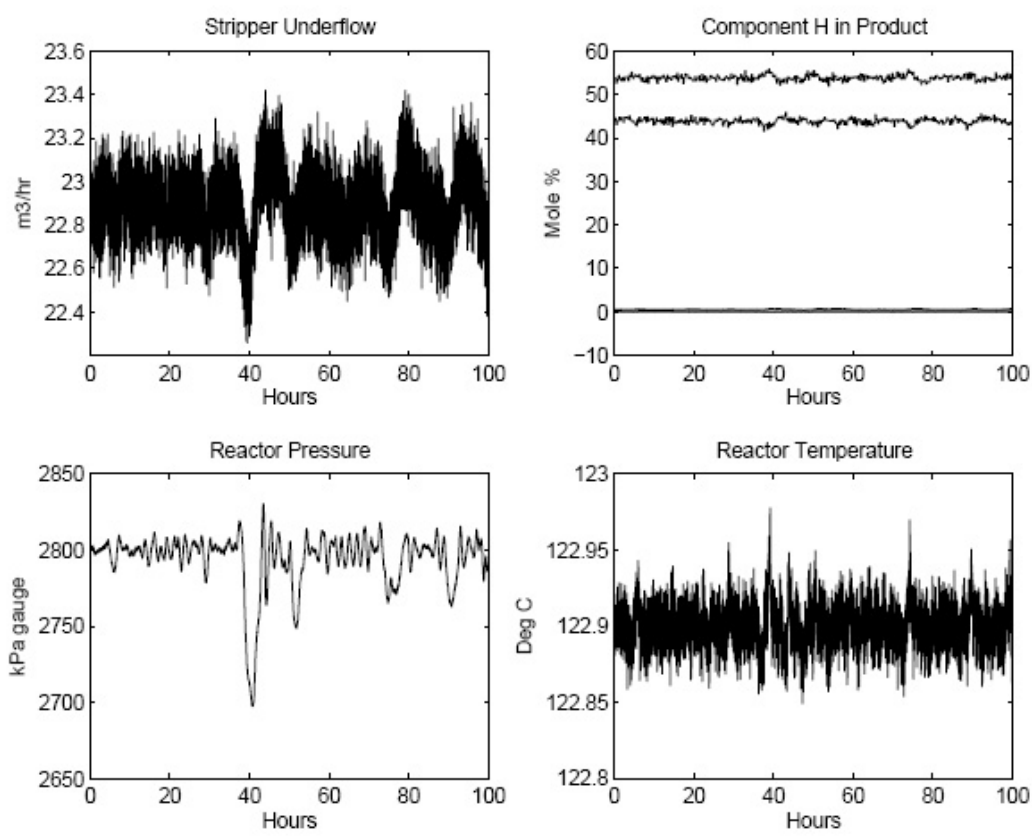

Figure 11. Closed loop response aga inst IDV(8) for the control design case by Ricker (1996) (author's nominal c ontroller pa ra meters). 

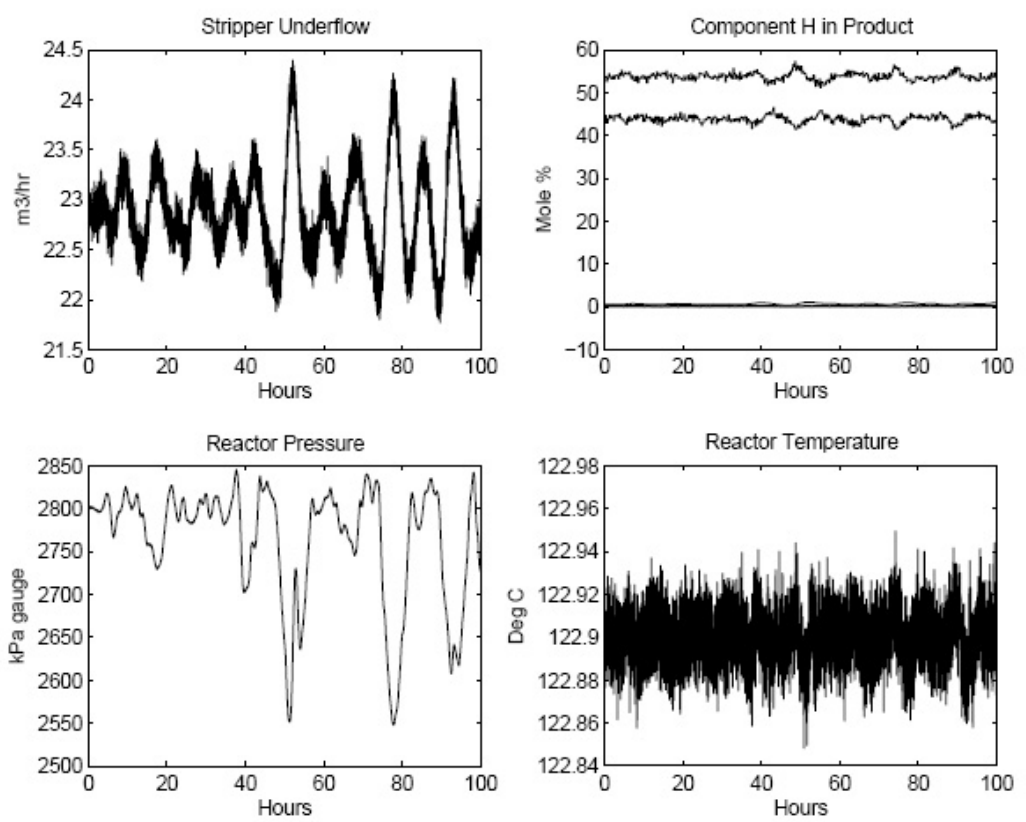

Figure 12. Closed loop response aga inst IDV(8) for the control design case by Ricker (1996) using optimal para meters $v_{\text {mits }}$.
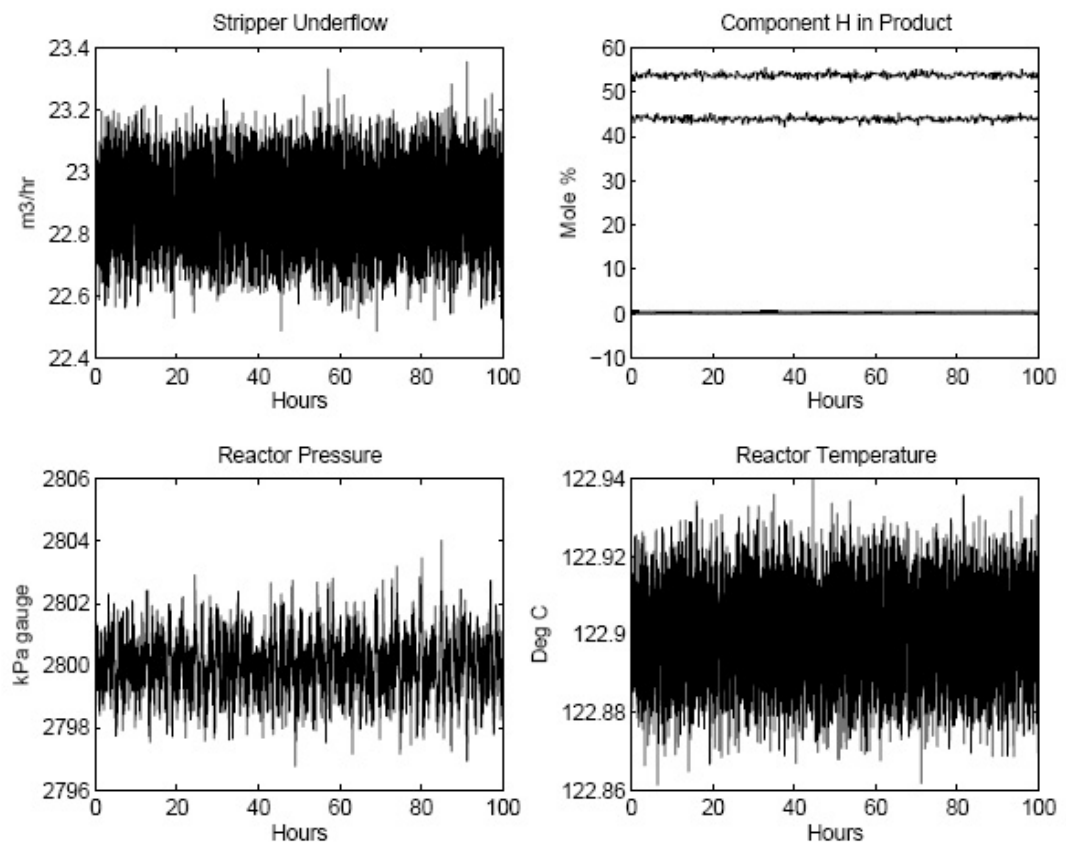

Figure 13. Closed loop response aga inst IDV(5) for the control design case by Ricker (1996) (author's nominal c ontroller pa ra meters). 

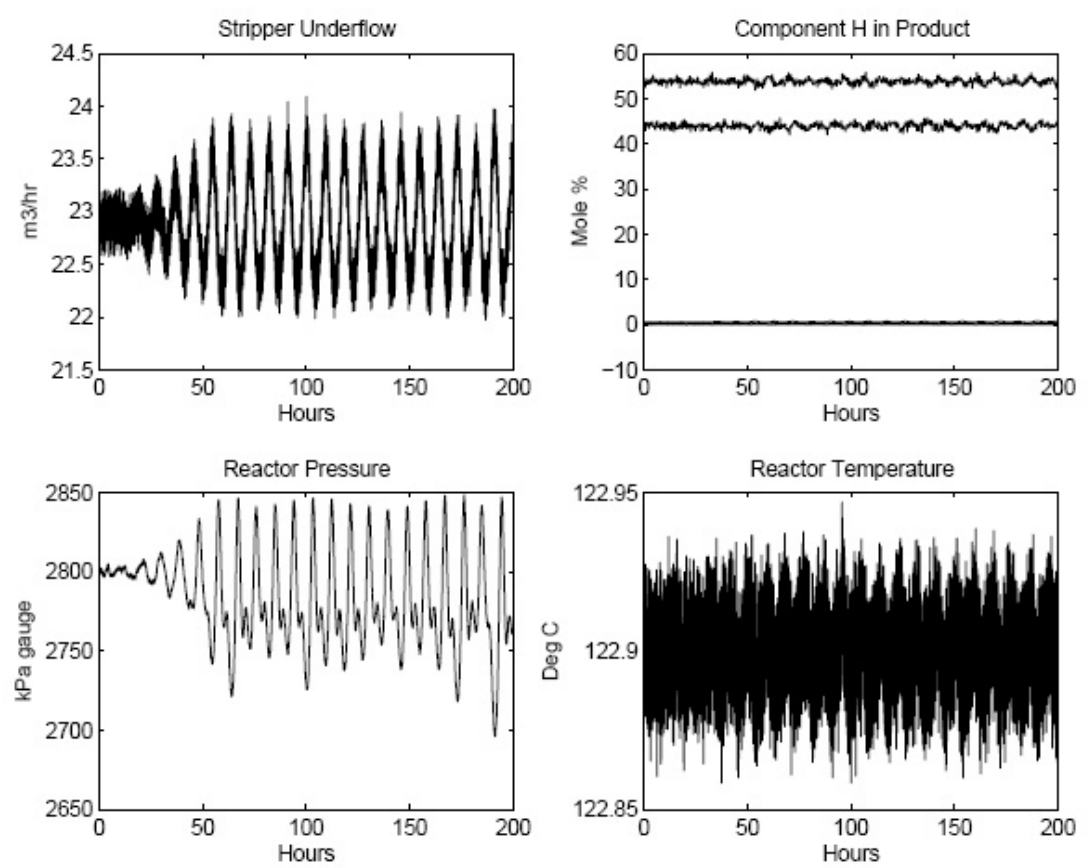

Figure 14. Closed loop response aga inst IDV(5) for the control design case by Ric ker (1996) using optimal para meters $v_{\text {mits }}$.

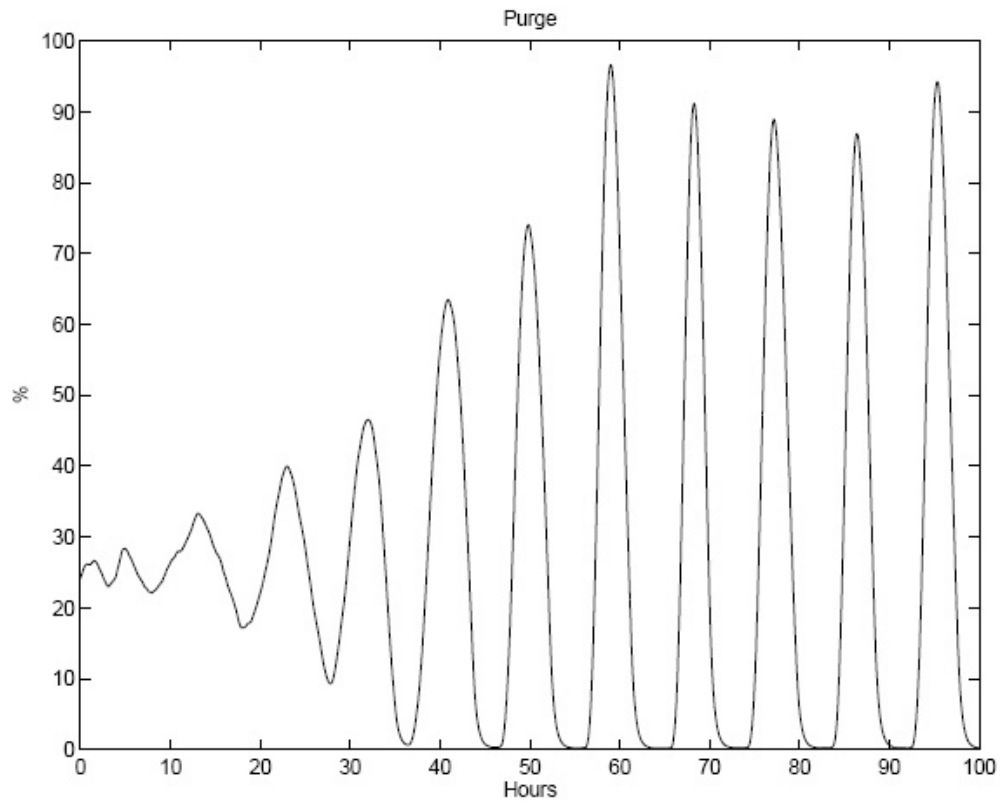

Figure 15. Purge valve position for the closed loop system (MIS optimal para meters) aga inst IDV(5) for Ric ker's control struc ture. 

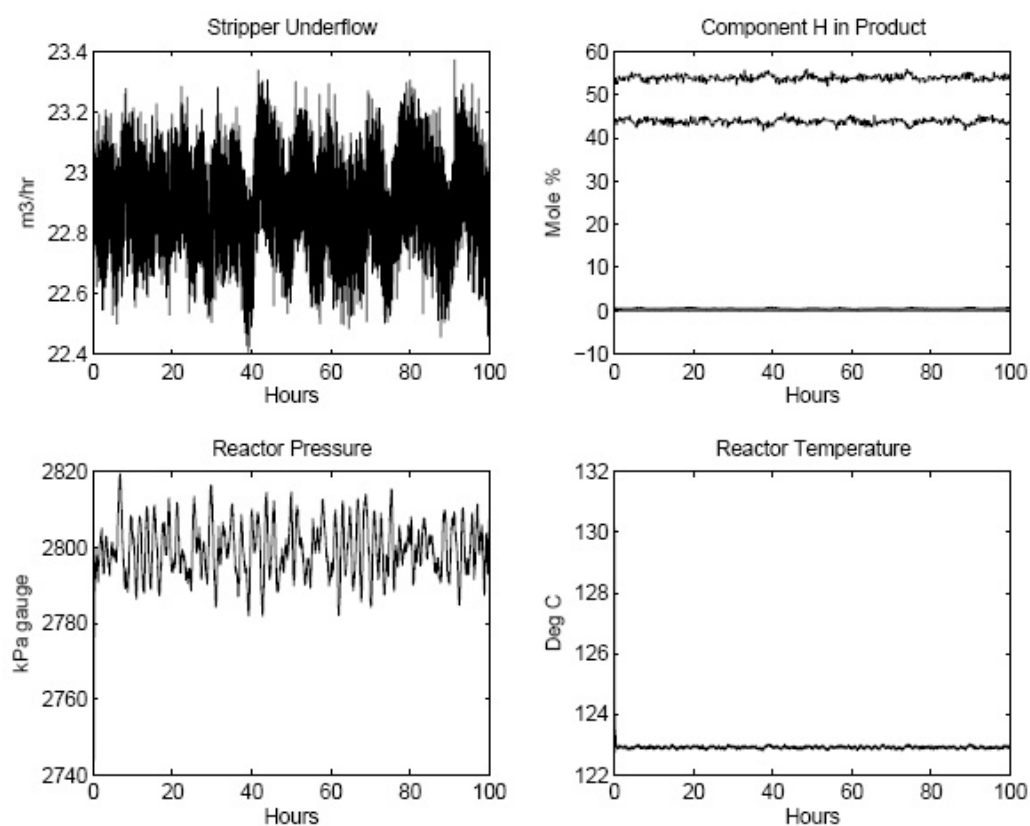

Figure 16. Closed loop response against IDV(8) for the control design case by Larsson et al. (2001) (authors' nominal controller pa ra meters).
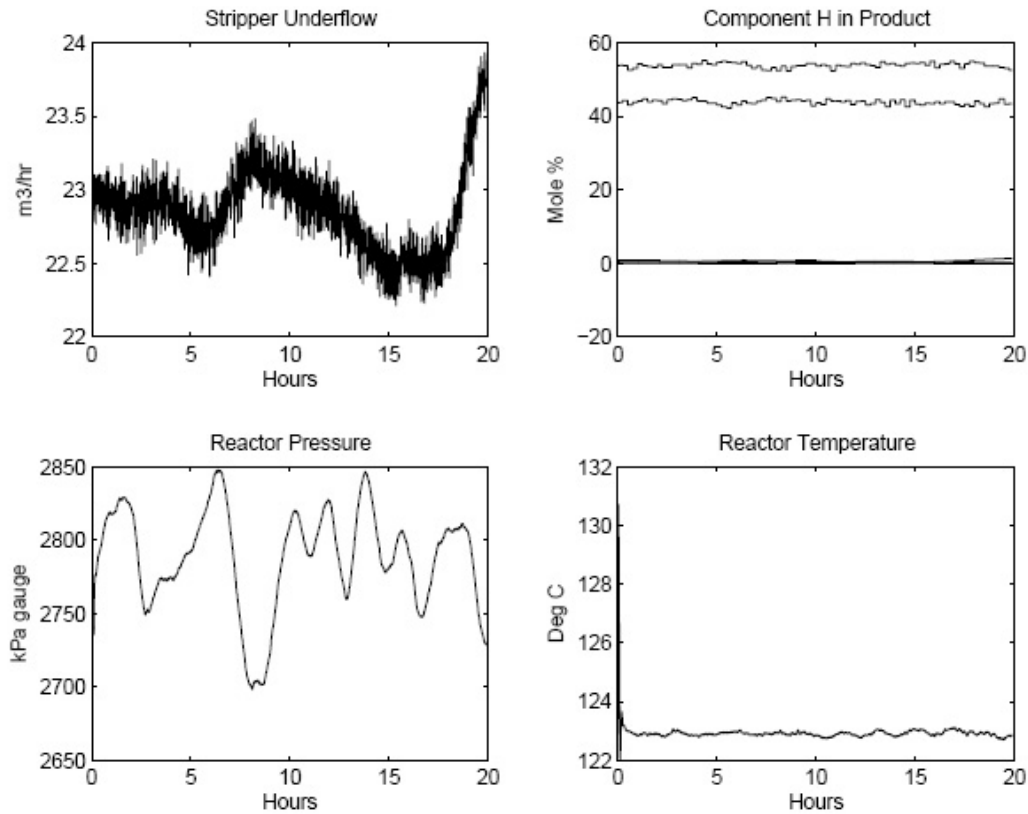

Figure 17. Closed loop response against IDV(8) for the control design case by Larsson et al. (2001) using optimal parameters $v_{\text {mits }}$. 

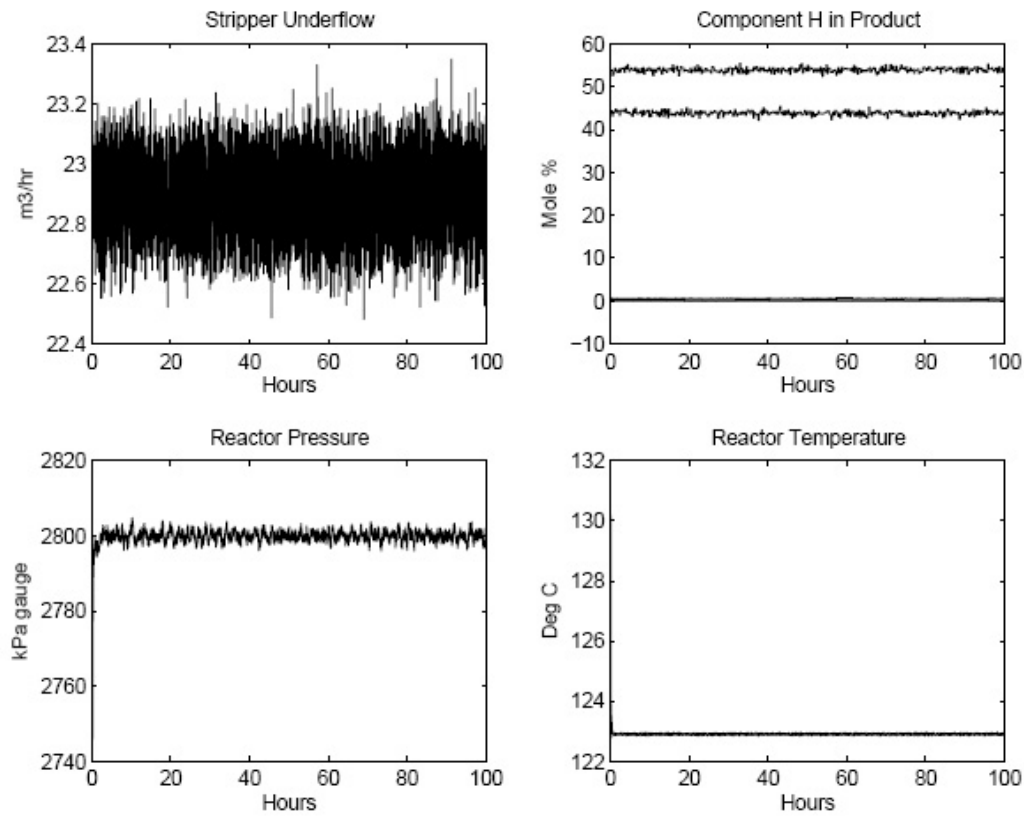

Figure 18. Closed loop response against IDV(5) for the control design case by Larsson et al. (2001) (authors' nominal controller pa rameters).
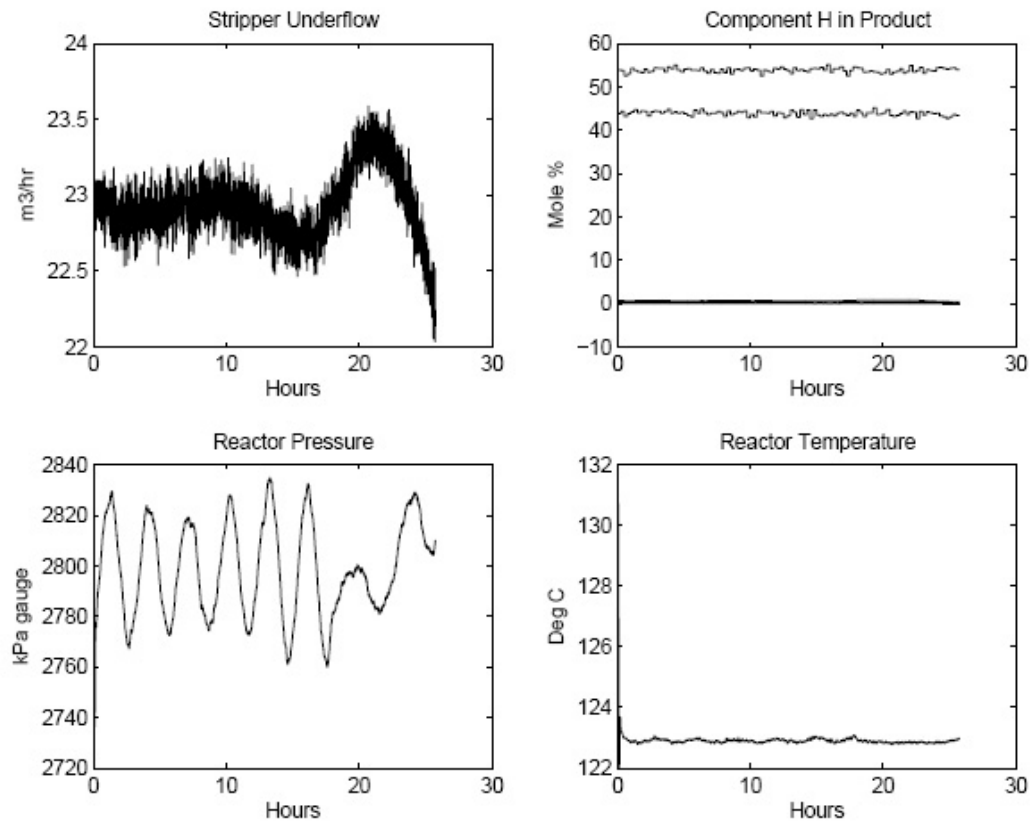

Figure 19. Closed loop response against IDV(5) for the control design case by Larsson et al. (2001) using optimal para meters $v_{\text {mits }}$. 

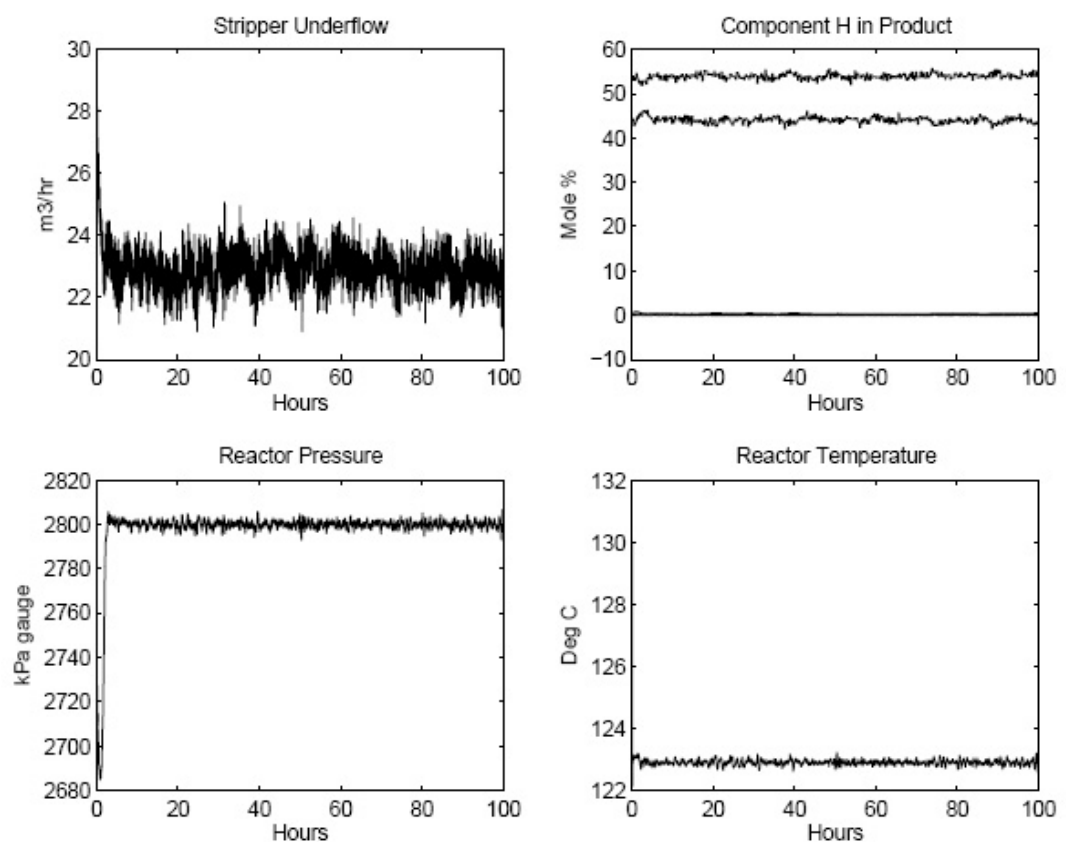

Figure 20. Closed loop response against IDV(8) for the proposed TBC design (authors' nominal controller parameters).
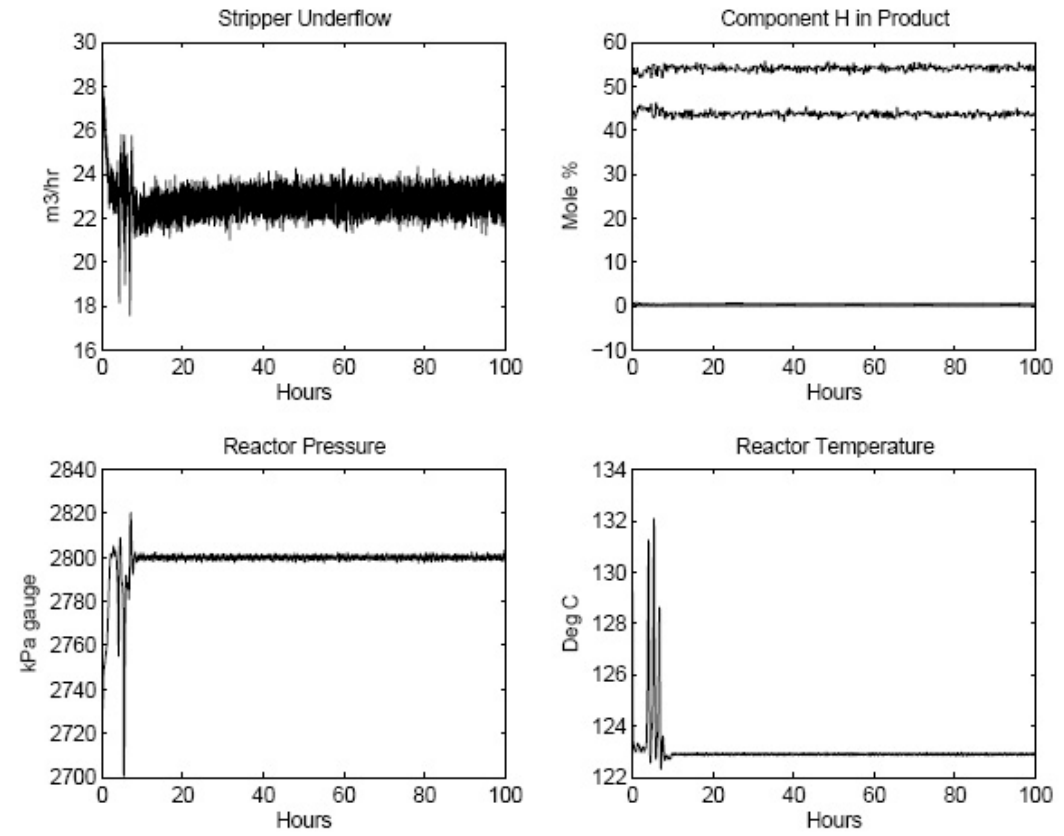

Figure 21. Closed loop response against IDV(8) for the proposed TBC design using optimal parameters $v_{\text {mits }}$. 

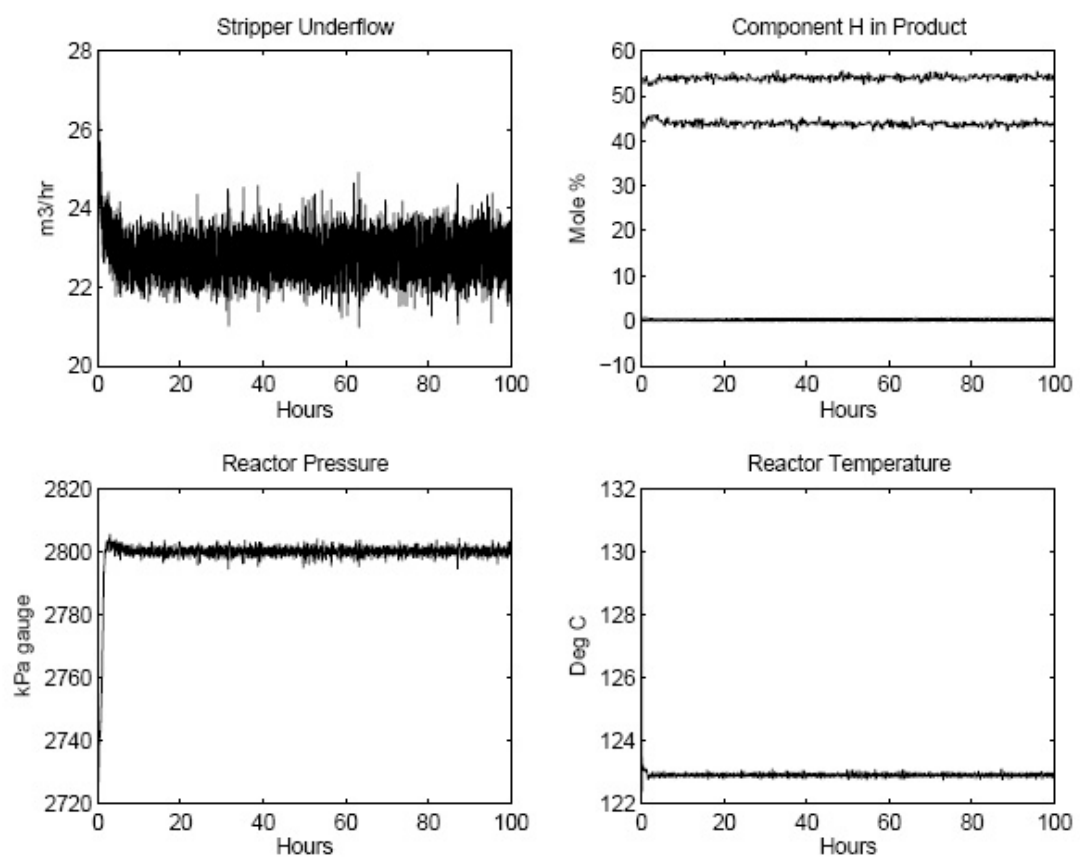

Figure 22. Closed loop response against IDV(5) for the proposed TBC design (authors' nominal controller parameters).
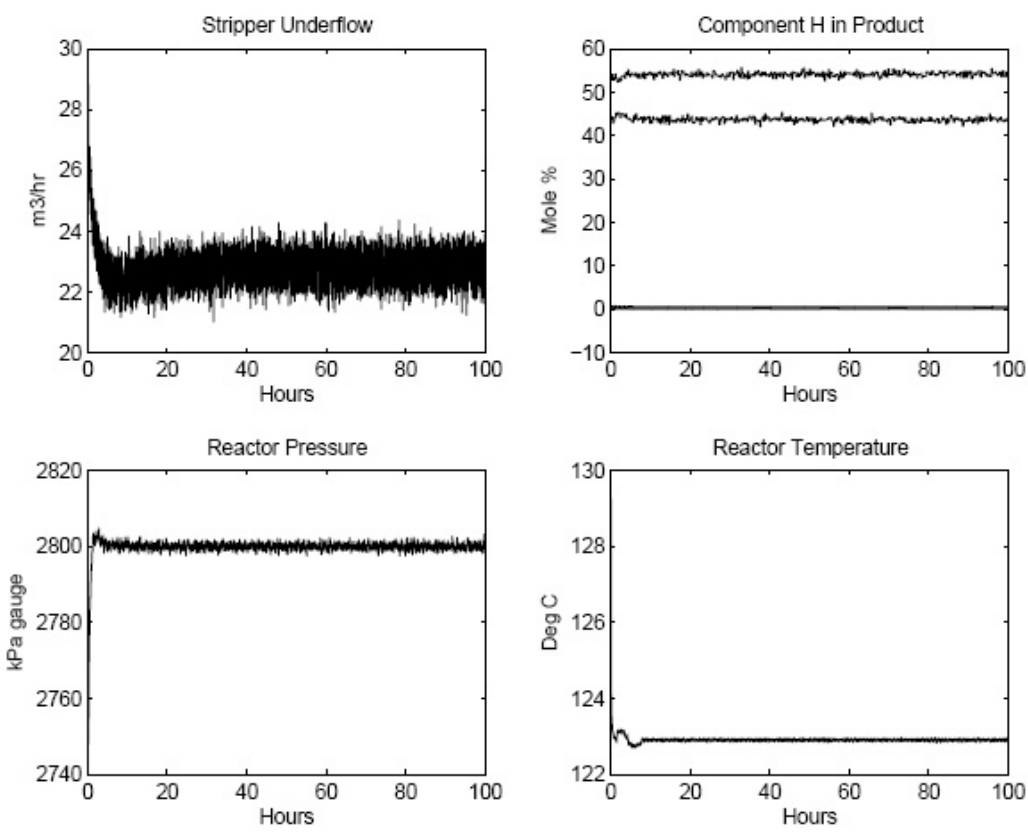

Figure 23. Closed loop response against IDV(5) for the proposed TBC design using optimal parameters $v_{\text {mits }}$. 

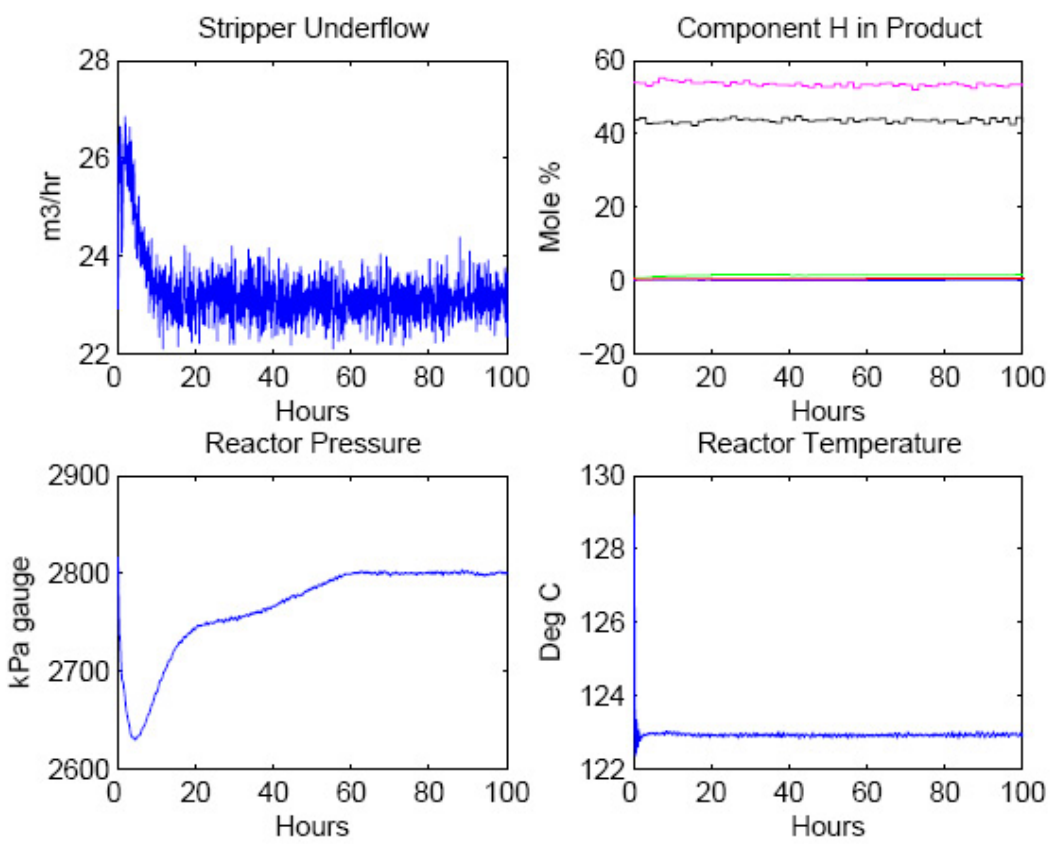

Figure 24. Closed loop response against IDV(5) for the cost improved TBC design using optimal parameters $v_{\text {mits }}$.

Table 1. Optimal controller parameters for Ricker's control structure

\begin{tabular}{|c|c|c|c|c|c|c|c|c|c|}
\hline $\mathrm{v}$ & Control Loop & $\mathrm{v}_{0}$ & $v_{\text {nomad }}$ & $\mathrm{v}_{\text {mits }}$ & $\mathrm{v}$ & Control Loop & $\mathrm{v}_{0}$ & $v_{\text {nomad }}$ & $\mathrm{v}_{\text {mits }}$ \\
\hline$v(1)$ & A Feed Flow & 0.01 & 0.005 & 0.0050113 & $v(20)$ & A Feed Flow & $1.6667 e^{-5}$ & $2.5 e^{-5}$ & $2.5 e^{-5}$ \\
\hline$v(2)$ & D Feed Flow & $1.6 e^{-6}$ & $8 e^{-7}$ & $8 e^{-7}$ & $v(21)$ & D Feed Flow & $1.6667 e^{-5}$ & $2.5 e^{-5}$ & $2.5 e^{-5}$ \\
\hline$v(3)$ & E Feed Flow & $1.8 e^{-6}$ & $2.7 e^{-6}$ & $2.7 e^{-6}$ & $v(22)$ & E Feed Flow & $1.6667 e^{-5}$ & $8.333 e^{-6}$ & $8.3358 e^{-6}$ \\
\hline$v(4)$ & C Feed Flow & 0.003 & 0.0045 & 0.0045 & $v(23)$ & C Feed Flow & $1.6667 e^{-5}$ & $8.333 e^{-6}$ & $8.3841 e^{-6}$ \\
\hline$v(5)$ & Putge Flow & 0.01 & 0.005 & 0.005022 & $v(24)$ & Purge Flow & $1.6667 e^{-5}$ & $1.7513 e^{-5}$ & $1.7722 e^{-5}$ \\
\hline$v(6)$ & Seporator Flow & 0.0004 & 0.0002 & 0.0002 & $v(25)$ & Separator Flow & $1.6667 e^{-5}$ & $2.5 e^{-5}$ & $2.5 e^{-5}$ \\
\hline$v(7)$ & Stripper Flow & 0.0004 & 0.0002 & 0.0002 & $v(26)$ & Stripper Flow & $1.6667 e^{-5}$ & $2.5 e^{-5}$ & $2.5 e^{-5}$ \\
\hline$v(8)$ & Production Rate & 3.2 & 4.8 & 4.8 & $v(27)$ & Production Rate & 2 & 1 & 1 \\
\hline$v(9)$ & Stripper Level & -0.0002 & -0.0003 & -0.0003 & $v(28)$ & Stripper Level & 3.3333 & 1.6667 & 1.6667 \\
\hline$v(10)$ & Separator Level & -0.001 & -0.0015 & -0.0015 & $v(29)$ & Separator Level & 3.3333 & 1.6667 & 1.6667 \\
\hline$v(11)$ & Reactor Level & 0.8 & 0.4 & 0.4 & $v(30)$ & Reactor Level & 1 & 1.1077 & 1.159 \\
\hline$v(12)$ & Reactor Pressure & -0.0001 & $-5 e^{-5}$ & $-5 e^{-5}$ & $v(31)$ & Reactor Pressure & 0.3333 & 0.5 & 0.5 \\
\hline$v(13)$ & $\% G$ in Product & -0.4 & -0.6 & -0.6 & $v(32)$ & \%G in Product & 1.6667 & 0.8333 & 0.8333 \\
\hline$v(14)$ & \%A to Reactor & 0.0002 & 0.0003 & 0.0003 & $v(33)$ & $\% A$ to Reactor & 1 & 1.5 & 1.5 \\
\hline$v(15)$ & $\% A / C$ to Reactor & 0.0003 & 0.00015 & 0.00015004 & $v(34)$ & $\% A / C$ to Reactor & 2 & 1 & 1.003 \\
\hline$v(16)$ & Reactor Temp. & -8 & -12 & -11.998 & $v(35)$ & Reactor Temp. & 0.125 & 0.0625 & 0.065701 \\
\hline$v(17)$ & Separator Temp. & -4 & -2 & -2 & $v(36)$ & Separator Temp. & 0.25 & 0.375 & 0.375 \\
\hline$v(18)$ & G/H Product Ratio & 32 & 16 & 16 & & & & & \\
\hline$v(19)$ & G/H Product Ratio & 46 & 69 & 69 & $J(8 / h)$ & & 115.80 & 105.565 & 105.580 \\
\hline
\end{tabular}

Table 1. Optimal c ontroller pa ra meters for Ric ker's control struc ture. 
Table 2. Optimal controller parameters for the control structure by Larsson et al. (2001)

\begin{tabular}{|c|c|c|c|c|c|c|c|c|c|}
\hline $\mathrm{v}$ & Control Loop & $\mathrm{v}_{0}$ & $\mathrm{v}_{\text {nomad }}$ & $\mathbf{v}_{\text {mits }}$ & $\mathrm{v}$ & Control Loop & $\mathrm{v}_{0}$ & $\mathrm{v}_{\text {nomad }}$ & $\mathrm{v}_{\text {mits }}$ \\
\hline$v(1)$ & A Feed Flow & 0.01 & 0.0010977 & 0.0011 & $v(20)$ & A Feed Flow & $1.6667 e^{-5}$ & $1.6667 e^{-5}$ & $9 e^{-6}$ \\
\hline$v(2)$ & D Feed Flow & $1.6 e^{-6}$ & $1.6 e^{-6}$ & $9.21287 e^{-8}$ & $v(21)$ & D Feed Flow & $1.6667 e^{-5}$ & $1.6667 e^{-5}$ & $1.8333 e^{-5}$ \\
\hline$v(4)$ & $C$ Feed Flow & 0.004 & 0.004732 & 0.00323 & $v(23)$ & C Feed Flow & $1.6667 e^{-5}$ & $1.6667 e^{-5}$ & $9 e^{-6}$ \\
\hline$v(5)$ & Purge Flow & 0.01 & 0.01 & 0.00972 & $v(24)$ & Purge Flow & $1.6667 e^{-5}$ & $1.6667 e^{-5}$ & $31.8004 e^{-5}$ \\
\hline$v(8)$ & Production Rate & 3.2 & 7.1375 & 7.1710 & $v(27)$ & Production Rate & 2 & 0.5625 & 0.5383 \\
\hline$v(9)$ & Stripper Level & -0.0002 & -0.000322 & -0.000259 & $v(28)$ & Stripper Level & 3.3333 & 1.3021 & 0.8375 \\
\hline$v(10)$ & Separator Level & $-0,001$ & $-0,000512$ & -0.00209 & $v(29)$ & Separator Level & 3.3333 & 3.0833 & 3 \\
\hline$v(11)$ & Reactor Level & 0.8 & 0.3 & 0.2269 & $v(30)$ & Reactor Level & 1 & 1.75 & 0.9 \\
\hline$v(12)$ & Reactor Pressure & -0.0001 & -0.0001 & $-6.165 e^{-6}$ & $v(31)$ & Reactor Pressure & 0.3333 & 0.14583 & 0.03 \\
\hline$v(16)$ & Reactor Temp. & -8 & -7.5 & -6.8392 & $v(35)$ & Reactor Temp. & 0.125 & 0.1602 & 0.1125 \\
\hline$v(17)$ & Separator Temp. & -4 & -7.3125 & -7.2616 & $v(36)$ & Separator Temp. & 0.25 & 0.5156 & 0.5225 \\
\hline$v(18)$ & G/H Product Ratio & 32 & 29 & 43.7854 & & & & & \\
\hline$v(19)$ & G/H Product Ratio & 46 & 17 & 53.3266 & $J(8 / h)$ & & 122.724 & 96.976 & 90.508 \\
\hline
\end{tabular}

Table 2. Optimal controller parameters for the control structure by Larsson et al.

(2001).

Table 3. Optimal controller parameters for the control structure by Antelo et al. (2006)

\begin{tabular}{|c|c|c|c|c|c|c|c|c|c|}
\hline $\mathrm{v}$ & Control Loop & $\mathrm{v}_{0}$ & $v_{\text {nomad }}$ & $v_{\text {mits }}$ & $\mathrm{v}$ & Control Loop & $\mathrm{v}_{0}$ & $v_{\text {nomad }}$ & $\mathbf{v}_{\text {mits }}$ \\
\hline$v(1)$ & A Feed Flow & 0.001 & 0.0015 & 0.0015 & $v(20)$ & A Feed Flow & $1.6667 e^{-5}$ & $1.6667 e^{-5}$ & $8.333 e^{-6}$ \\
\hline$v(2)$ & D Feed Flow & 0.003 & 0.0045 & 0.003679 & $v(21)$ & D Feed Flow & $1.6667 e^{-5}$ & $8.333 e^{-6}$ & $8.333 e^{-6}$ \\
\hline$v(3)$ & E Feed Flow & $1.8 e^{-10}$ & $9.0527 e^{-11}$ & $1.78 e^{-10}$ & $v(22)$ & E Feed Flow & 4.1667 & 6.25 & 3.9482 \\
\hline$v(4)$ & CFeed Flow & 20 & 20 & 16.356 & $v(23)$ & C Feed Flow & 0.1667 & 2.5 & 1.3531 \\
\hline$v(5)$ & Condenser Coolant & $7 e^{-7}$ & $3.5547 e^{-7}$ & $5.742 e^{-7}$ & $v(24)$ & Condenser Coolant & 4.1667 & 6.25 & 3.2567 \\
\hline$v(6)$ & Separator Flow & $4 e^{-4}$ & 0.0006 & 0.0002002 & $v(25)$ & Separator Flow & $1.6667 e^{-5}$ & $1.5023 e^{-5}$ & $1.0116 e^{-5}$ \\
\hline$v(7)$ & Stripper Flow & 0.004 & 0.002 & 0.003957 & $v(26)$ & Stripper Flow & $1.6667 e^{-5}$ & $2.5 e^{-5}$ & $8.934 e^{-5}$ \\
\hline$v(8)$ & Production Rate & 3.2 & 4.8 & 1.6203 & $v(27)$ & Production Rate & 2 & 2 & 2.4541 \\
\hline$v(9)$ & Stripper Level & -0.02 & -0.02 & $-0,01591$ & $v(28)$ & Stripper Level & 0.3333 & 0.3333 & 0.3734 \\
\hline$v(10)$ & Separator Level & -0.05 & $-0,05$ & -0.03301 & $v(29)$ & Separator Level & 3.3333 & 3.125 & 4.7419 \\
\hline$v(11)$ & Reactor Level & 10 & 7.9102 & 6.5012 & $v(30)$ & Reactor Level & 0.01667 & 0.025 & 0.0161 \\
\hline$v(12)$ & Reactor Pressure & -0.0001 & -0.00015 & -0.0001135 & $v(31)$ & Reactor Pressure & 0.3333 & 0.17008 & 0.3654 \\
\hline$v(13)$ & \%G in Product & -0.032 & -0.048 & -0.02782 & $v(32)$ & $\% G$ in Product & 1.6667 & 1.3941 & 1.2395 \\
\hline$v(14)$ & \%A in Purge & 0.0009 & 0.00135 & 0.001064 & $v(33)$ & $\% A$ in Purge & 9.3667 & 9.3667 & 4.7380 \\
\hline$v(15)$ & Recycle Rate & 0.00125 & 0.001875 & 0.001875 & $v(34)$ & Recycle Rate & 25 & 12.5 & 12.5 \\
\hline$v(16)$ & Reactor Temp. & -8 & -8 & -10.5164 & $v(35)$ & Reactor Temp. & 0.125 & 0.125 & 0.1641 \\
\hline$v(17)$ & Separator Temp. & 100 & 50 & 104.34 & $v(36)$ & Separator Temp & 8.3333 & 8.333 & 10,2088 \\
\hline$v(18)$ & G/H Product Ratio & 32 & 31.996 & 30.244 & & & & & \\
\hline$v(19)$ & G/H Product Ratio & 46 & 69 & 34.4868 & $J(8 / h)$ & & 156.843 & 148.745 & 147.587 \\
\hline
\end{tabular}

Table 3. Optimal controller parameters for the control struc ture by Antelo et al.

(2007b). 
Table 4. Optimal tuning for the $T B C$ structure (Figure 7)

\begin{tabular}{|c|c|c||c|c|c|}
\hline $\mathbf{v}$ & Control Loop & $\mathbf{v}_{\mathbf{m i t s}}$ & $\mathbf{v}$ & Control Loop & $\mathbf{v}_{\mathbf{m i t s}}$ \\
\hline$v(1)$ & A Feed Flow & $1.4029 e^{-3}$ & $v(20)$ & A Feed Flow & $1.584 e^{-5}$ \\
\hline$v(2)$ & D Feed Flow & 0.004043 & $v(21)$ & D Feed Flow & $1.4535 e^{-5}$ \\
\hline$v(3)$ & E Feed Flow & $9.6882 e^{-11}$ & $v(22)$ & E Feed Flow & 3.1266 \\
\hline$v(4)$ & C Feed Flow & 30.0 & $v(23)$ & C Feed Flow & 0.83334 \\
\hline$v(5)$ & Condenser Coolant & $9.8858 e^{-7}$ & $v(24)$ & Condenser Coolant & 2.0834 \\
\hline$v(6)$ & Separator Flow & $4.5727 e^{-4}$ & $v(25)$ & Separator Flow & $1.42116 e^{-5}$ \\
\hline$v(7)$ & Stripper Flow & 0.004242 & $v(26)$ & Stripper Flow & $1.302 e^{-5}$ \\
\hline$v(8)$ & Production Rate & 4.8 & $v(27)$ & Production Rate & 1.2995 \\
\hline$v(9)$ & Stripper Level & -0.021864 & $v(28)$ & Stripper Level & 0.19213 \\
\hline$v(10)$ & Separator Level & -0.048376 & $v(29)$ & Separator Level & 1.6704 \\
\hline$v(11)$ & Reactor Level & 8.5024 & $v(30)$ & Reactor Level & 0.011874 \\
\hline$v(12)$ & Reactor Pressure & $-5 e^{-5}$ & $v(31)$ & Reactor Pressure & 0.18142 \\
\hline$v(13)$ & \%G in Product & -0.02811 & $v(32)$ & \% G in Product & 1.0927 \\
\hline$v(14)$ & \%A in Purge & $5.664^{-4}$ & $v(33)$ & \%A in Purge & 10.284 \\
\hline$v(15)$ & Recycle Rate & 0.001271 & $v(34)$ & Recycle Rate & 30.338 \\
\hline$v(16)$ & Reactor Temp. & -8.1636 & $v(35)$ & Reactor Temp. & 0.06475 \\
\hline$v(17)$ & Separator Temp. & 90.219 & $v(36)$ & Separator Temp & 6.4701 \\
\hline$v(18)$ & G/H Product Ratio & 21.762 & & & \\
\hline$v(19)$ & G/H Product Ratio & 30.891 & $J(\$ / h)$ & & 84.289 \\
\hline
\end{tabular}

Table 4. Optimal tuning for the TBC structure (Figure 7).

Table 5. Objective function values for all the control structures and disturbances in a time horizon of $t=100 \mathrm{~h}$

\begin{tabular}{|c|c|c|c|c|}
\hline Control Case & Disturbance & Original Value & NOMADm Value & MITS Value \\
\hline \multirow{2}{*}{ Ricker } & IDV(5) & 114.102 & $117.956(3.38 \%)$ & $117.958(3.38 \%)$ \\
\cline { 2 - 5 } Larsson et al. & IDV(8) & 118.275 & $126.578(7.02 \%)$ & $126.582(7.02 \%)$ \\
\cline { 2 - 5 } & IDV(5) & 114.032 & $113.598(-0.38 \%)$ & $115.510(1.30 \%)$ \\
\hline \multirow{2}{*}{$T B C$} & IDV (8) & 114.284 & $128.113(12.10 \%)$ & $122.615(7.29 \%)$ \\
\cline { 2 - 5 } & IDV (5) & 150.879 & $153.395(1.66 \%)$ & $154.083(2.12 \%)$ \\
\hline Improved $T B C$ & IDV $(5)$ & 149.639 & $153.382(2.5 \%)$ & $153.879(2.83 \%)$ \\
\hline
\end{tabular}

Table 5. Objective function values for all the control structures and disturbances in a time horizon of $\mathrm{t}=100 \mathrm{~h}$. 